\title{
The effects of individualised intermittent theta burst stimulation in the prefrontal cortex: A TMS-EEG study
}

\author{
Sung Wook Chung ${ }^{1} \odot$ | Caley M. Sullivan ${ }^{1} \mid$ Nigel C. Rogasch ${ }^{2} \mid$ Kate E. Hoy ${ }^{1}$ | \\ Neil W. Bailey ${ }^{1}$ | Robin F. H. Cash ${ }^{1}$ | Paul B. Fitzgerald ${ }^{1,3}$
}

${ }^{1}$ Monash Alfred Psychiatry Research Centre, Monash University, Central Clinical School and The Alfred, Melbourne, Australia

${ }^{2}$ Brain and Mental Health Laboratory, School of Psychological Sciences and Monash Biomedical Imaging, Monash Institute of Cognitive and Clinical Neuroscience, Monash University, Melbourne, Australia

${ }^{3}$ Epworth Clinic, Epworth Healthcare, Melbourne, Australia

\section{Correspondence}

Sung Wook Chung, Monash Alfred Psychiatry Research Centre, Level 4, 607 St Kilda Road, Melbourne, VIC 3004, Australia.

Email: sungwook.chung@monash.edu

Funding information

National Health and Medical Research Council; NHMRC Practitioner Fellowship, Grant/Award Number: 1078567; NHMRC Career

Development Fellowship, Grant/Award Number: 1082894; NHMRC Early Career Fellowship, Grant/Award Number: 1072057;

Monash Graduate Scholarship

\begin{abstract}
Recent studies have highlighted variability in response to theta burst stimulation (TBS) in humans. TBS paradigm was originally developed in rodents to mimic gamma bursts coupled with theta rhythms, and was shown to elicit long-term potentiation. The protocol was subsequently adapted for humans using standardised frequencies of stimulation. However, each individual has different rhythmic firing pattern. The present study sought to explore whether individualised intermittent TBS (Ind iTBS) could outperform the effects of two other iTBS variants. Twenty healthy volunteers received iTBS over left prefrontal cortex using $30 \mathrm{~Hz}$ at $6 \mathrm{~Hz}, 50 \mathrm{~Hz}$ at $5 \mathrm{~Hz}$, or individualised frequency in separate sessions. Ind iTBS was determined using thetagamma coupling during the 3-back task. Concurrent use of transcranial magnetic stimulation and electroencephalography (TMS-EEG) was used to track changes in cortical plasticity. We also utilised mood ratings using a visual analogue scale and assessed working memory via the 3-back task before and after stimulation. No group-level effect was observed following either 30 or $50 \mathrm{~Hz}$ iTBS in TMS-EEG. Ind iTBS significantly increased the amplitude of the TMS-evoked P60, and decreased N100 and P200 amplitudes. A significant positive correlation between neurophysiological change and change in mood rating was also observed. Improved accuracy in the 3-back task was observed following both $50 \mathrm{~Hz}$ and Ind iTBS conditions. These findings highlight the critical importance of frequency in the parameter space of iTBS. Tailored stimulation parameters appear more efficacious than standard paradigms in neurophysiological and mood changes. This novel approach presents a promising option and benefits may extend to clinical applications.
\end{abstract}

\section{KEYWORDS}

mood, prefrontal cortex, theta burst stimulation (TBS), theta-gamma coupling, TMS-EEG, working memory

\section{1 | INTRODUCTION}

Theta burst stimulation (TBS) is a modified form of repetitive transcranial magnetic stimulation (rTMS) which is able to modulate brain activity beyond the time of stimulation in humans (Huang, Edwards, Rounis, Bhatia, \& Rothwell, 2005). TBS was originally developed from the observation of patterned neuronal firing that occurred in rats during exploratory behaviour (Larson \& Munkacsy, 2015). The stimulation pattern mimicking such bursts of neuronal firing, that is, the combination of the complex-spike pattern (gamma frequency at $100 \mathrm{~Hz}$ ) with a theta frequency $(\sim 5 \mathrm{~Hz})$ repetition rate, resulted in robust long-term potentiation (LTP) in the hippocampal slices (Larson, Wong, \& Lynch, 1986). This patterned stimulation protocol was adapted in humans using similar frequency parameters to animal models and has been widely used for over a decade. Typically, TBS in humans involves the application of high-frequency bursts $(3$ pulses at $50 \mathrm{~Hz}$ ) at lowfrequency interval $(5 \mathrm{~Hz})$ using a total of 600 pulses at $70-80 \%$ of active/resting motor threshold (a/rMT). When applied continuously (cTBS) for $40 \mathrm{~s}$, TBS has shown to decrease corticospinal excitability measured via motor-evoked potentials (MEPs) for up to $60 \mathrm{~min}$. When applied intermittently (iTBS; $2 \mathrm{~s}$ on, $8 \mathrm{~s}$ off) for $192 \mathrm{~s}$, an opposite effect was observed up to $30 \mathrm{~min}$ (Huang et al., 2005). 
Despite early reports of robust changes in the size of MEPs beyond the stimulation duration (Di Lazzaro et al., 2008; Huang et al., 2005), studies of TBS have shown large variability in recent years. Studies with larger sample sizes have shown no overall effects of TBS (Hamada, Murase, Hasan, Balaratnam, \& Rothwell, 2013; LopezAlonso, Cheeran, Rio-Rodriguez, \& Fernandez-Del-Olmo, 2014), and a recent meta-analysis has found evidence that the effect sizes in the literature may be overestimated (Chung, Hill, Rogasch, Hoy, \& Fitzgerald, 2016). One possible reason for the large variability in responses to TBS may be due to the direct adaptation of the method used in the animal studies. The peak frequency of theta oscillations not only differs between rodents and humans (Jacobs, 2014; Watrous et al., 2013), but also between subjects and within subjects at different time points (Klimesch, Doppelmayr, Russegger, \& Pachinger, 1996). Some studies have modified the frequency of TBS and found more robust effects in the motor region (Goldsworthy, Pitcher, \& Ridding, 2012) and frontal eye fields (FEF) (Nyffeler et al., 2006a, b). While it remains unknown which frequency is responsible for the enhanced outcome, targeting the centre frequency of the intrinsic rhythm, that is, $6 \mathrm{~Hz}$ in theta waves $(4-8 \mathrm{~Hz})$, may have played an important role. More recently, Brownjohn, Reynolds, Matheson, Fox, and Shemmell (2014) investigated whether applying TBS at individual theta peak would result in larger effects in the motor cortex, however, improved effects were not obtained compared with conventional TBS. It is possible that the interaction between modulating (theta) and modulated (gamma) signals is more important for improving the effect of TBS. The relationship between theta and gamma, also known as theta-gamma coupling (TGC), plays a key role in cognitive processing and communication between brain regions (Lisman, 2010; Lisman \& Jensen, 2013; Schack, Vath, Petsche, Geissler, \& Moller, 2002; Tort, Komorowski, Manns, Kopell, \& Eichenbaum, 2009). In humans, TGC has been observed during working memory tasks in hippocampal intracranial (Chaieb et al., 2015) and electroencephalography (EEG) recordings (Friese et al., 2013; Koster, Friese, Schone, Trujillo-Barreto, \& Gruber, 2014; Park, Jhung, Lee, \& An, 2013). Given that the theta-gamma relationship is variable between subjects, TGC may hold the key to improving the effects of TBS using more physiologically derived parameters.

The optimisation of TBS by tailoring the protocol at individual level would have potential clinical importance as TBS is increasingly being investigated as an alternative to conventional rTMS in various clinical populations due to its short application time and low intensity requirement (Desmyter et al., 2016; Prasser et al., 2015; Turriziani et al., 2012). The variability in neurophysiological and behavioural outcomes present therapeutic limitations, an obstacle that needs to be addressed. In particular, research should address this issue by examining clinically relevant areas such as the prefrontal cortex, the focus of investigation for psychiatric and cognitive disorders.

Advances in technology have facilitated the measurement of plastic changes following neuromodulation in non-motor regions using concurrent recording of TMS and EEG (TMS-EEG) (Casula, Pellicciari, Ponzo, et al., 2016b; Chung et al., 2017; Chung, Rogasch, Hoy, \& Fitzgerald, 2015; Hill, Rogasch, Fitzgerald, \& Hoy, 2017). Measuring TMS-evoked responses before and after neuromodulatory paradigms provides a metric of neural plasticity at the cortical level. For instance, a positive peak at a latency of $60 \mathrm{~ms}$ (P60) may provide a marker of excitability in motor and prefrontal regions (Cash, Noda, et al., 2017c; Hill et al., 2017), whereas a negative peak at a latency of $100 \mathrm{~ms}$ (N100) may be associated with $\mathrm{GABA}_{\mathrm{B}}$-mediated inhibitory mechanisms [in motor regions (Bonnard, Spieser, Meziane, de Graaf, \& Pailhous, 2009; Premoli, Rivolta, et al., 2014b; Rogasch, Daskalakis, \& Fitzgerald, 2013a); in prefrontal regions (Chung et al., 2017; Rogasch, Daskalakis, \& Fitzgerald, 2015)], and these two peaks have been the most consistent neuromodulatory-mediated effects observed in recent literature using prefrontal TMS-EEG (Casula, Pellicciari, Picazio, Caltagirone, \& Koch, 2016a; Chung, Rogasch, Hoy, Sullivan, et al., 2018b; Hill et al., 2017). Consequently, the balance in the relationship between the P60 and $\mathrm{N} 100$ has been proposed to relate to the balance of neural excitation and inhibition in humans (Noda, Zomorrodi, Cash, et al., 2017d), which is a quintessential property of neural function, and its importance in modulating behavioural function has been demonstrated in animal (Yizhar et al., 2011) and human studies (Lisman, 2012).

Increased interest in prefrontal application of TBS has also led to the investigation of associated cognitive processes. Evidence suggests TBS over the prefrontal cortex may also modulate executive functioning in healthy individuals (Cho et al., 2010; Verbruggen, Aron, Stevens, \& Chambers, 2010; Viejo-Sobera et al., 2017). iTBS to the left prefrontal cortex has been shown to improve n-back task performances (Hoy et al., 2016; Viejo-Sobera et al., 2017), while cTBS had the opposite effect (Lee \& D'Esposito, 2012; Schicktanz et al., 2015; Viejo-Sobera et al., 2017). Furthermore, a recent meta-analysis has demonstrated that the effectiveness of iTBS may be task-dependent and most reliable effects were observed in working memory paradigms (Lowe, Manocchio, Safati, \& Hall, 2018). Therefore, it is plausible that the after-effects of prefrontal iTBS can be quantified via modulation of $n$-back task performance.

In the present study, we investigated the effects of different frequencies of $\mathrm{iTBS}(30 \mathrm{~Hz}$ at $6 \mathrm{~Hz}, 50 \mathrm{~Hz}$ at $5 \mathrm{~Hz}$, and individualised frequency) on neurophysiological measures using TMS-EEG over the left prefrontal cortex. We also measured mood on a visual analogue scale (VAS) and working memory performance via 3-back task following iTBS to investigate relationship between the neurobiological effects of iTBS and the change in behaviour. Due to the implications of P60 and N100 in cortical plasticity mentioned above and the frequent observation of the change in the amplitude of these peaks following neuromodulatory paradigms over the left prefrontal cortex (Chung, Rogasch, Hoy, Sullivan, et al., 2018b; Hill et al., 2017; Noda, Zomorrodi, Backhouse, et al., 2017c), we hypothesised that individualised iTBS would produce the strongest change in these components. We also anticipated $30 \mathrm{~Hz}$ iTBS to be superior to $50 \mathrm{~Hz}$ stimulation given the robustness seen in the motor cortex study (Chung et al., 2016; Goldsworthy et al., 2012).

\section{2 | MATERIAL AND METHODS}

\section{1 | Participants}

Twenty right-handed healthy subjects ( $26.0 \pm 9.2$ years, 13 female) volunteered in the study. The average years of education were 
$16.5 \pm 3.0$ years. All participants were screened with Mini International Neuropsychiatric Interview to confirm no history of psychiatric illness (Sheehan et al., 1998). Participants reported no history of neurological illness or brain injury, were non-smokers and free of psychoactive drugs including over-the-counter medications. Participants had also refrained from consumption of caffeinated drinks and alcohol for at least $24 \mathrm{hr}$ prior to the experiment. Apart from two subjects, the time of day of the experiments (morning/afternoon) was kept constant across sessions. Written informed consent was obtained from al study participants. Ethics approval for the study was obtained from the Alfred Hospital and Monash University Human Research and Ethics Committee.

\subsection{Procedure}

Figure 1 depicts the overview of the experimental design. Each participant attended three sessions (pseudorandomised) with each session at least $72 \mathrm{hr}$ apart to avoid any potential carry-over effects. The experimental procedures comprised concurrent recording of EEG during 75 single TMS pulses at baseline (BL), 5 -min post (T5) and $30-$ min post (T30) iTBS over the left prefrontal cortex. Volunteers received iTBS at varying frequency; either (1) $30 \mathrm{~Hz}$ bursts repeated at $6 \mathrm{~Hz}$ (30 Hz iTBS), (2) $50 \mathrm{~Hz}$ bursts repeated at $5 \mathrm{~Hz}$ (50 Hz iTBS) or (3) individualised frequency (Ind iTBS) in each session. Subjects also performed the 3-back working memory task at BL, 20-min post (T20) and 45-min post (T45) iTBS while EEG was recording. Participants rated their current mood on visual analogue scales (VAS) at BL and 60-min post (T60) iTBS. During the resting period at BL, theta-gamma coupling (TGC) from the EEG data during the 3-back task was analysed off-line to determine individualised iTBS stimulation frequencies (see below for details).

\section{3 | EEG recordings}

EEG recordings were obtained from 50 TMS-compatible $\mathrm{Ag} / \mathrm{AgCl}$ electrodes on a 64-channel EEG cap (FP1, FP2, AF3, AF4, F7, F5, F3,
F1, Fz, F2, F4, F6, F8, FC5, FC3, FC1, FCz, FC2, FC4, FC6, T7, C5, C3, C1, Cz, C2, C4, C6, T8, CP5, CP3, CP1, CP2, CP4, CP6, P7, P5, P3, P1, $\mathrm{Pz}, \mathrm{P} 2, \mathrm{P} 4, \mathrm{P} 6, \mathrm{P} 8, \mathrm{PO} 3, \mathrm{PO}, \mathrm{PO} 4, \mathrm{O} 1, \mathrm{Oz}, \mathrm{O} 2$ ) via Synamps ${ }^{2}$ amplifier onto Neuroscan Acquire software (Compumedics, Melbourne, Australia). Electrodes were on-line referenced to $\mathrm{CPz}$ and grounded to FPz. For TMS-EEG recordings, EEG signals were amplified (1,000x) and low-pass filtered $(\mathrm{DC}-2,000 \mathrm{~Hz})$ with a high acquisition rate of $10,000 \mathrm{~Hz}$ using a large operating window $( \pm 200 \mathrm{mV}$ ). For EEG recordings during the 3-back task, EEG signals were filtered $(0.05-200 \mathrm{~Hz})$ and sampled at $1,000 \mathrm{~Hz}$ with an operating range of $\pm 950 \mu \mathrm{V}$. During TMS-EEG recordings, participants listened to white noise through intra-auricular earphones (Etymotic Research, ER3-14A, USA) to limit the contamination of the EEG signals produced by the TMS click sound (Nikouline, Ruohonen, \& Ilmoniemi, 1999; Rogasch et al., 2014). The sound level was adjusted individually until single TMS pulses at $120 \%$ rMT were adequately blocked.

\section{4 | Transcranial magnetic stimulation}

Both single-pulse TMS and iTBS were delivered using a figure-of-eight MagVenture B-65 fluid-cooled coil (MagVenture A/S, Denmark) in a biphasic mode. Stimuli were applied to the left hemisphere with the coil positioned at $45^{\circ}$ angle relative to midline (handle pointing posterior). The EEG cap was applied first, and then the resting motor threshold (rMT) was determined (i.e., by applying TMS over the cap) as the minimum stimulus intensity required to elicit at least three out of six motor evoked potentials (MEPs) $>0.05 \mathrm{mV}$ in amplitude (Conforto, Z'Graggen, Kohl, Rosler, \& Kaelin-Lang, 2004) in the relaxed first dorsal interosseous muscles. Prefrontal TMS was administered over F1 electrode as previously described (Chung, Rogasch, Hoy, \& Fitzgerald, 2018a; Chung, Rogasch, Hoy, Sullivan, et al., 2018b). This electrode sits over the superior frontal gyrus (BA 6, 8 and 9) (Koessler et al., 2009) which is part of left dorsolateral prefrontal cortex (DLPFC). The edge of the coil was marked on the cap for consistent re-positioning of the coil. This has shown accuracy to within $5 \mathrm{~mm}$ when neuronavigation is not available (Rogasch,

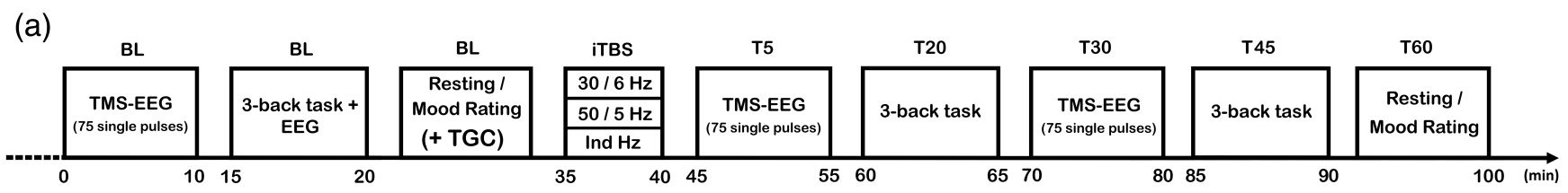

(b)

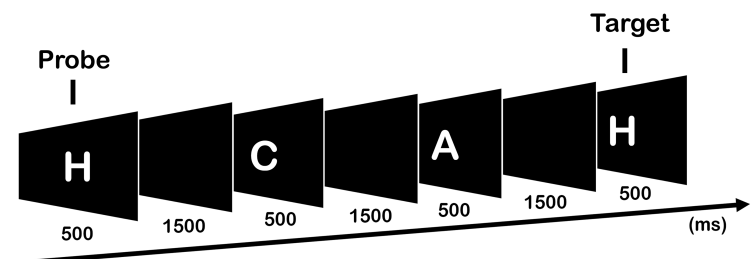

(c)

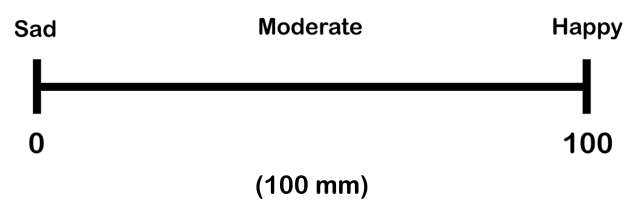

FIGURE 1 Schematic diagram of the experimental design of the study. (a) Combined transcranial magnetic stimulation and electroencephalography (TMS-EEG), 3-back task and mood rating were completed at baseline (BL). Theta-gamma coupling (TGC) was analysed off-line using the EEG data from the 3-back task. Intermittent theta burst stimulation (iTBS) was administered at one of three frequency patterns ( $30 \mathrm{~Hz}$ every $6 \mathrm{~Hz}, 50 \mathrm{~Hz}$ every $5 \mathrm{~Hz}$ and individualised Hz). TMS-EEG was repeated at T5 and T30, the 3-back task at T20 and T45 and mood rating at T60. (b) An example of a correctly responded trial for the 3-back task. Subjects were instructed to remember each stimulus and respond with a button press when the presented letter corresponded to the one that appeared 3 letters before. (c) Visual analogue scale (VAS) rating. Subjects drew a line on a $100 \mathrm{~mm}$ VAS to indicate their current mood, from sad (0) to happy (100) 
Thomson, Daskalakis, \& Fitzgerald, 2013b). The TMS coil was handheld, and a thin plastic template was mounted on the EEG cap to ensure $45^{\circ}$ angle and tangential placement of coil and to further improve the consistency within and between sessions (Supporting Information Material, Section S1). Subjects received 75 single pulses ( $5 \mathrm{~s}$ interval $\pm 10 \%$ jitter) to the left prefrontal cortex at $120 \% \mathrm{rMT}$ before and after different iTBS conditions; (1) $30 \mathrm{~Hz}$ bursts repeated at $6 \mathrm{~Hz}$ (Goldsworthy et al., 2012), (2) $50 \mathrm{~Hz}$ bursts repeated at $5 \mathrm{~Hz}$ (Huang et al., 2005), (3) individualised frequency. Each iTBS block consisted of a burst of 3 pulses repeated 10 times with an $8 \mathrm{~s}$ break for a total of 600 pulses. The intensity of stimulation was adjusted to $75 \%$ of individuals' rMT. This intensity was selected as our previous study demonstrated more robust cortical effects following iTBS compared with $50 \%$ or $100 \%$ rMT (Chung, Rogasch, Hoy, Sullivan, et al., 2018b). The average intensity for each condition was as follows (mean \pm SD): $30 \mathrm{~Hz} \quad \mathrm{iTBS}=51.6 \pm 6.5 \% ; \quad 50 \mathrm{~Hz} \quad \mathrm{iTBS}=51.6 \pm 6.4 \% ; \quad$ Ind iTBS $=51.6 \pm 6.4 \%$.

\section{5 | Working memory task}

Each participant performed 5 min of the 3-back task before $(B L)$ and after (T20 and T45) iTBS. A randomised series of white letters (A-J) were presented consecutively on a black screen for $500 \mathrm{~ms}$ followed by 1,500 ms of a blank screen. Participants were instructed to remember each stimulus and press a button when the presented letter corresponded to the one that appeared three letters earlier (3-back) (Figure 1b). The task contained 25\% target trials out of 130 letters in total. Working memory performance was evaluated using the $d$ prime sensitivity ( $d^{\prime} ; z$-transformed values of hit-rate minus false-alarm rate) and accurate reaction time (Haatveit et al., 2010).

\section{6 | Mood rating}

The mood rating was assessed via self-rated visual analogue scale (VAS) (Ahearn, 1997), which has been used to evaluate the mood state in both clinical (Le-Niculescu et al., 2009) and healthy populations (Robinson, Cools, Crockett, \& Sahakian, 2010; Robinson \& Sahakian, 2009). Subjects drew a line on a $100 \mathrm{~mm}$ VAS to indicate their current mood compared with saddest subject has ever felt (0) and happiest subject has ever felt (100) (Figure 1c).

\section{7 | Manipulation of the pulse intervals}

For a flexible and rapid manipulation of pulse intervals for iTBS, the Arduino open-source microcontroller platform was used (https:// www.arduino.cc/). The Arduino is an inexpensive, low-level microcontroller which has an excellent temporal resolution owing to its property of bypassing the hardware and software environments of modern operating systems (D'Ausilio, 2012). Several studies have shown that the Arduino is able to measure signals with less than $1 \mathrm{~ms}$ variability (D'Ausilio, 2012; Schubert, D'Ausilio, \& Canto, 2013; Schultz \& van Vugt, 2016), making it an ideal low-cost lab equipment. A customised script allowed for an instantaneous manipulation of pulses at desired theta and gamma frequencies. This process reduced the waiting period for the manual programming of MagVenture machine and ensured subject blinding and consistent procedural steps across different conditions. A comparison example between the MagVenture and Arduino programmed stimuli $(50 \mathrm{~Hz}$ iTBS) can be found in the Supporting Information Material, which shows no difference between the two techniques in TBS trigger timing, Figure S2.

\section{8 | Selection of individualised frequencies of iTBS based on theta-gamma coupling}

The individualised frequency for Ind iTBS was determined by the phase-amplitude cross-frequency coupling (PAC) between frontal theta (phase) and parietal gamma (amplitude) oscillations during the 3-back task. The rationale for using working memory task EEG rather than resting EEG for the individualisation was: (1) to mimic the original TBS study in mice where the stimulation protocol was derived from the patterns of neuronal firing which occurred during learning or exploratory behaviour (Larson et al., 1986); (2) to minimise the variance of individuals' state by actively engaging in the same task for each subject, in contrast to resting EEG recordings during which participants could be performing a wide variety of neural functions and (3) the implication of theta-gamma coupling in working memory which may serve as a biomarker of behavioural performance (Axmacher et al., 2010; Park et al., 2013; Tamura, Spellman, Rosen, Gogos, \& Gordon, 2017; Tort et al., 2009).

Detailed information on individualisation procedure can be found in Supporting Information Material, Section S3. Briefly, 10 correct trials were selected by randomly ordering the epochs and using the first 10 epochs after shuffling for TGC (45 s in length; Figure 2a). The raw signals were filtered at the respective frequencies; $3-9 \mathrm{~Hz}$ for theta ( $F z$ electrode) and $20-70 \mathrm{~Hz}$ for gamma (Pz electrode). Data were subjected to Hilbert transform and theta-filtered gamma amplitude envelope was then extracted prior to PAC estimation (Figure 2b). Phase-amplitude coupling between theta and gamma was calculated using a general linear model (GLM) (Penny, Duzel, Miller, \& Ojemann, 2008) and performed at every filter step to produce a comodulogram matrix. The peak of the comodulogram matrix was used to infer the specific frequencies within the theta $(4-8 \mathrm{~Hz})$ and gamma $(30-60 \mathrm{~Hz})$ bands at which the highest coupling occurred, yielding individual theta and gamma frequencies for iTBS (Figure 2c shows examples from two participants, maximum value indicated by black asterisks). Participants' individualised frequency of stimulation are plotted in Figure 2d, with an average of gamma frequency at $41.90 \pm 7.7 \mathrm{~Hz}$ and theta frequency at $5.97 \pm 1.0 \mathrm{~Hz}$. This procedure was performed for every condition to be consistent across different sessions and thereby minimising any potential differences in total duration of the experiment.

\section{9 | EEG data preprocessing}

TMS-EEG data were analysed offline using EEGLAB (Delorme \& Makeig, 2004), TESA (Rogasch et al., 2017), FieldTrip (Oostenveld, Fries, Maris, \& Schoffelen, 2011) toolboxes and custom scripts within the MATLAB platform (R2015b, The MathWorks, USA). Preprocessing steps of EEG data followed previous description (Chung et al., 2017). Data were epoched around the TMS pulse $(-1,000$ to $1,000 \mathrm{~ms})$, baseline corrected ( -500 to $-50 \mathrm{~ms}$ ) and the large magnetic pulses 
(a)

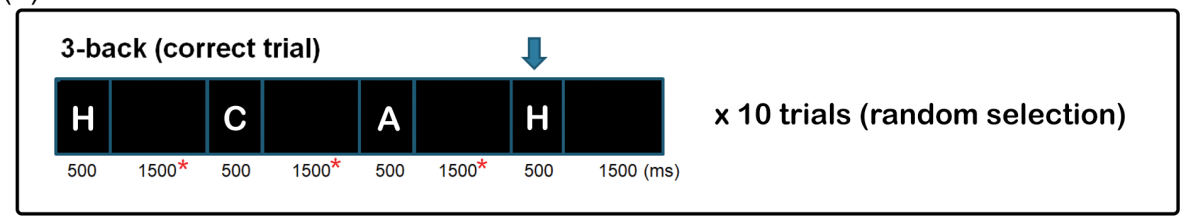

(b)

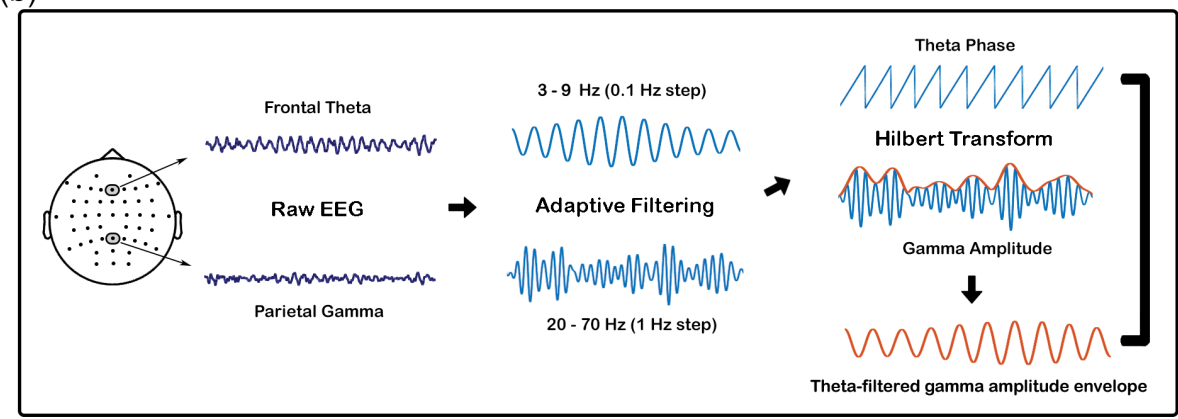

(c)

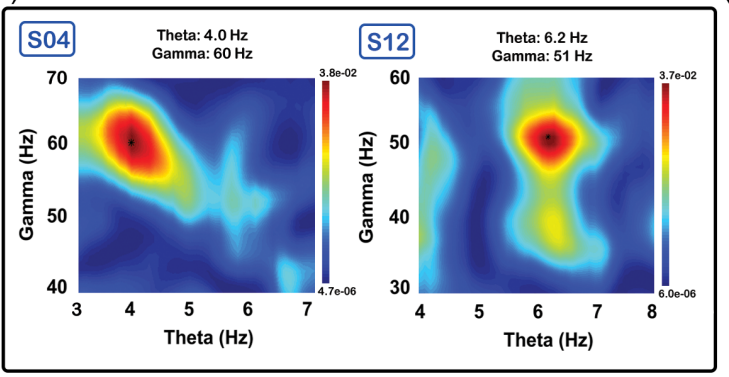

(d)

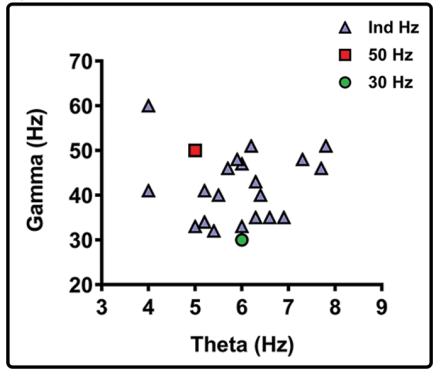

FIGURE 2 Procedures involved in the selection of the individualised frequency of iTBS. (a) Ten random correct trials selected for theta-gamma coupling (TGC). Only data from the maintenance period of each epoch (red asterisks) were included in the calculation to avoid spurious coupling resulting from the visual-evoked response and edge effects. (b) Raw data from Fz electrode was chosen as the modulating frequency (theta), and Pz electrode as the modulated frequency (gamma). Data were filtered (adaptive) in the frequency range in multiple steps and Hilbert transform was applied to obtain phase (theta) and amplitude (gamma). Theta-filtered gamma amplitude envelope was extracted prior to phase-amplitude coupling estimation using general linear model. (c) Comodulogram illustrated for two subjects (S04 and S12). Peak was detected using the maximum value in the frequency ranges of interest (4-8 Hz for theta, 30-60 Hz for gamma). (d) Values used for individualised iTBS in blue triangle, $50 \mathrm{~Hz}$ iTBS in red rectangle, and $30 \mathrm{~Hz}$ iTBS in green circle [Color figure can be viewed at wileyonlinelibrary.com]

were removed and interpolated ( -5 to $15 \mathrm{~ms}$ ). The epoched data were concatenated across three time points (BL, T5 and T30) to avoid bias in component rejection from the independent component analysis (ICA). Data were downsampled to $1,000 \mathrm{~Hz}$ and manual inspection was performed to remove epochs containing excessive noise (i.e., burst of muscle activity) and/or disconnected electrodes. The average number of epochs included in the analyses for each condition was as follows (mean $\pm S D$ ): $30 \mathrm{~Hz}$ iTBS $=73.0 \pm 3.2 ; 50 \mathrm{~Hz}$ iTBS = $72.4 \pm 3.6$; Ind iTBS $=73.5 \pm 2.1$. Two rounds of ICA (FastICA, "tanh" contrast) were performed for the artefact rejection using semiautomated component classification algorithm (tesa_compselect function; Rogasch et al., 2017). The first ICA was used to remove the remaining tail of TMS-evoked muscle artefacts (Rogasch et al., 2014) which was identified if the component time course was eight times larger than the mean absolute amplitude across the entire time course. All data were band-pass (Butterworth, second-order, zero-phase, 1-80 Hz) and band-stop filtered (line noise removal, 49-51 Hz), and epochs were visually inspected again to remove any anomalous activity in the EEG data. The second round of ICA was performed to remove other non-neural artefacts including eye blinks and saccadic movement (mean absolute $z$ score of two frontal electrodes FP1 and
FP2 $>2.5$ ), persistent muscle activity (high frequency power $>60 \%$ of total power), decay artefact and electrode noises (absolute $z$ score of an electrode[s] $>4$ ). Removed channels were interpolated and FP1 and FP2 were removed from all the datasets as these channels were generally contaminated by artefacts. Finally, data were re-referenced to common average and were segregated into original time point blocks (BL, T5 and T30) and epochs averaged.

For the EEG data during the 3-back task, continuous EEG data were band-pass (Butterworth, second-order, zero-phase, 0.1-80 Hz) and band-stop (49-51 Hz) filtered. Data were then epoched around the correctly responded trial $(-7,000$ to $1,000 \mathrm{~ms})$ which contained a correct probe (e.g., first " $\mathrm{H}$ " in Figure 2a), correct holds (correctly not responded; e.g., "D" and "E" in Figure 2a) and a correct response (e.g., " $\mathrm{H}$ " with an arrow above in Figure 2a). This epoch was chosen for having all sequence of items leading to a correct response. Data were baseline corrected to the entire trial, visually inspected to remove any epochs containing a burst of muscle activity and underwent one round of ICA. The same component rejection was performed as the second round of ICA of TMS-EEG data. Any removed channels were then interpolated, FP1 and FP2 removed as mentioned above, and data were re-referenced to common average. 


\subsection{0 | TMS-evoked potentials}

Graphical representation of the waveforms was produced using the average of three fronto-central electrodes (FC1, FCz and FC2) for the close proximity of the stimulation site (F1 electrode), while F1 was omitted to avoid introducing TMS coil contact related noise to the waveform (Rogasch, Daskalakis, et al., 2013a). Statistical analyses were conducted on TMS-evoked potentials (TEPs) using cluster-based permutation tests at a global scalp level. Comparisons were made using the averaged amplitude values of pre-defined time windows for the peaks of interest; N45 (40-55 ms), P60 (55-85 ms), N100 (95-135 ms) and P200 (160-240 ms). These peaks are commonly observed following prefrontal stimulation (Chung et al., 2017; Hill et al., 2017; Rogasch et al., 2014). Extraction of TEP values (for graphical representation and statistical/correlational analyses) was performed using the averaged signal \pm 5 ms of maximum (for positive peaks) and minimum (for negative peaks) values within the range window as above, consistent with previous studies (Chung et al., 2017; Hill et al., 2017; Opie, Rogasch, Goldsworthy, Ridding, \& Semmler, 2017). Studies have conducted correlational analyses between the modulations of TEPs and behaviour in attempts to better characterise neural processes involved in behavioural changes (Noda, Barr, Zomorrodi, Cash, Farzan, et al., 2017a; Rogasch, Daskalakis, et al., 2013a). The balance between excitation and inhibition may be more critical to neural function and behaviour than either neural excitability or inhibition alone (Bonansco \& Fuenzalida, 2016; Cash, Udupa, et al., 2017d; Meunier, Chameau, \& Fossier, 2017; Turrigiano, 2011), and evidence suggests TBS may have an influence in this regulatory property in animal (Moreau, Amar, Callebert, \& Fossier, 2013) and humans (Chung, Rogasch, Hoy, Sullivan, et al., 2018b; Hoy et al., 2016; Legon et al., 2016). Therefore, exploratory analyses were performed regarding the relationship between iTBS-induced change in P60 and N100 amplitudes in order to examine whether significant differences in any of the iTBS conditions could reflect altered inhibitory/excitatory balances (Noda, Zomorrodi, Cash, et al., 2017d).

\subsection{1 | Source estimation}

All estimation of the cortical source was performed using Brainstorm (Tadel, Baillet, Mosher, Pantazis, \& Leahy, 2011) which is documented and freely available for download online under the GNU general public licence (http://neuroimage.usc.edu/brainstorm/). Individual magnetic resonance imaging scans were unavailable, and hence EEG data were co-registered with the template model (ICBM 152). The forward model used the Symmetric Boundary Element Method implemented in OpenMEEG software (Gramfort, Papadopoulo, Olivi, \& Clerc, 2010) and the inverse model used the computation of minimum norm estimations (MNEs) with dipole orientations constrained to be normal to the cortex (Lin, Belliveau, Dale, \& Hamalainen, 2006). Differences in estimation were calculated using absolute subtraction.

\subsection{2 | Statistical analyses}

All statistical analyses were performed in SPSS (IBM Corp, Armonk, NY; Version 22), MATLAB, and FieldTrip. Analyses of TEPs were conducted using non-parametric cluster-based permutation statistics which provides a model-free method that does not run the risk of violating the assumptions of parametric tests and is an effective method of controlling for multiple comparisons across space (EEG channels) and time (Oostenveld et al., 2011). It is therefore commonly used in the analysis of TMS-EEG, EEG, MEG and MRI research (Casula, Pellicciari, Ponzo, et al., 2016b; Maris and Oostenveld, 2007; Opie et al., 2017; Premoli et al., 2017). Comparisons were first made across time point for each iTBS condition (within-comparison; between $\mathrm{BL}$ and T5/T30). Between-condition comparisons were performed using change-from-baseline scores (post-pre; $\Delta$ ). Monte Carlo $p$-values were calculated on 2,500 random permutations and clusters were defined as more than two neighbouring electrodes with a $p$-value of $<.05$, controlling for multiple comparisons across space $(p<.025$; two-tailed test).

For mood rating, one-way repeated measure analysis of variance (ANOVAs) were computed between conditions using $\Delta$ values (postpre). For the 3-back task, two-way repeated measure analysis of variance (ANOVAs) was used to investigate working memory performance 3 [stimulation conditions $(30 \mathrm{~Hz}, 50 \mathrm{~Hz}$, and Ind iTBS)] $\times$ 3 [time (BL, T20 and T45)]. Post-hoc pairwise comparisons were performed using Bonferroni corrections to further explore the significant main effects, while significant interactions were examined using oneway ANOVAs and paired $t$-tests.

For variability and correlational analyses, data were extracted from the average of six prefrontal electrodes (F1, Fz, F2, FC1, FCz and FC2). The TEP peaks were detected within the pre-defined time window as stated in Section 2.10 [N45 (40-55 ms), P60 (55-85 ms), $\mathrm{N} 100$ (95-135 ms) and P200 (160-240 ms)] and the amplitude was calculated by averaging the signal between $\pm 5 \mathrm{~ms}$ of the selected peak latency as previously described (Chung et al., 2017). Pearson's correlations were used to examine the relationship between the change in physiological measures (e.g., $\Delta \mathrm{N} 100$ ) and the change in behavioural outcome (e.g., $\Delta$ mood). The ratio between $\Delta \mathrm{N} 100$ and $\Delta$ $\mathrm{P} 60$ resulted in extreme outliers. The data were tested for normality (Shapiro-Wilk test) and outliers were winsorised by setting extreme values to the corresponding adjacent 5 th and 95th percentile value (Wilcox, 1997).

Post-hoc power analyses were performed to determine if the current study had enough power to detect the differences between conditions using G*Power (Version 3.1) software (Faul, Erdfelder, Lang, \& Buchner, 2007), which calculates the power $(1-\beta)$ as a function of $\alpha$ (0.05), the population effect size and the sample sizes (Cohen, 1988). G*power is a freely available software, and the details of the equations, tutorials and examples are available in the literature (Faul, Erdfelder, Buchner, \& Lang, 2009; Faul et al., 2007) and online (http:// www.gpower.hhu.de/). In addition, post-hoc Bayesian analysis was conducted to provide a more precise probability estimate of the results. Bayesian methods use prior probability distribution and the likelihood of the data to produce a posterior probability distribution (Dienes, 2016). The use of $p$-value null-hypothesis significance testing potentially portrays a binary view of statistical inference in which an observed effect is either real or the null hypothesis must be true (Stern, 2016). The Bayesian approach has the advantage in that it determines the likelihood of the observed data under each hypothesis from an equal perspective, and the statistical evidence can be more 
meaningfully quantified via Bayes factor (Biel \& Friedrich, 2018; Wagenmakers, 2007). The Bayesian analyses were conducted using JASP (Version 0.8.6.0) software (Wagenmakers, Love, et al., 2018a), an open-source project that is freely available online (https://jaspstats.org/). Bayes' rule is used to determine the Bayes factor, which is the change from prior to posterior odds inferred by the data (Wagenmakers, Marsman, et al., 2018b). Details of the equation, tutorials and examples can be found in the literature (Wagenmakers, Love, et al., 2018a; Wagenmakers, Marsman, et al., 2018b) and online.

\section{3 | RESULTS}

\section{1 | Baseline single-pulse TMS}

Single-pulse TMS over left prefrontal cortex resulted in a series of negative and positive peaks including N45, P60, N100 and P200 (Figure 3a). Consistent with other TMS-EEG studies in the prefrontal cortex (Chung et al., 2017; Hill et al., 2017; Rogasch et al., 2014), each peak showed a distinctive pattern in scalp topography (Figure 3b) and source estimation (Figure 3c).

\section{2 | Plastic effects of iTBS on TEPs}

We first performed comparisons on the amplitude of TEPs between stimulation conditions at BL, and found no significant differences (all $p>$.025; two-tailed test). We next assessed the iTBS-induced effects on the amplitude of TEPs within each stimulation condition over time (T5 vs. BL and T30 vs. BL). Testing for an effect in each peak of interest in pre-defined latency range (refer to Section 2.10), the clusterbased permutation tests revealed no significant differences between baseline (BL) and any of post-iTBS (T5 and T30) following both $30 \mathrm{~Hz}$ (Figure 4a) and $50 \mathrm{~Hz}$ (Figure 4b) iTBS at any peak (all $p>.025$ ).

However, Ind iTBS showed significant differences between BL and T5 for the P60 (increase, $p=.021$, frontal), N100 (decrease, $p=.014$ fronto-central) and P200 (decrease, $p=.012$, fronto-central; $p=.009$, posterior), and between T30 and BL for the P60 (increase, $p=.020$, posterior) and N100 (decrease, $p=.008$, fronto-central) (Figure 4c).

Examination of the response to each iTBS condition in TEPs demonstrated large inter-individual variability following both 30 and $50 \mathrm{~Hz}$ iTBS (see Table 1). More in-depth exploration of interindividual variability can be found in Supporting Information Material, Section S5.

We next conducted across-condition comparisons using the change-from-baseline scores $(\Delta)$ obtained from subtracting pre-signals (BL) from post-signals (T5 and T30). We found that $\Delta \mathrm{P} 60$ was significantly larger following Ind iTBS compared with $30 \mathrm{~Hz}$ iTBS at T30 ( $p=.018$, frontal) (Figure 5a), and compared with $50 \mathrm{~Hz}$ iTBS at T5 ( $p=.021$, frontal; $p=.015$, posterior) and T30 ( $p=.022$, posterior) (Figure $5 b$ ). Source estimation of P60 largely corroborated the results of the sensor-level analysis whereby Ind iTBS showed increased current density at the site of stimulation while minimal changes were seen following $30 \mathrm{~Hz}$ or $50 \mathrm{~Hz}$ iTBS (Figure 5c). iTBS-induced change in other peaks ( $\Delta$ N45, $\Delta$ N100 and $\Delta$ P200) yielded in no significant differences in these comparisons (all $p>.025$ ). In addition, no

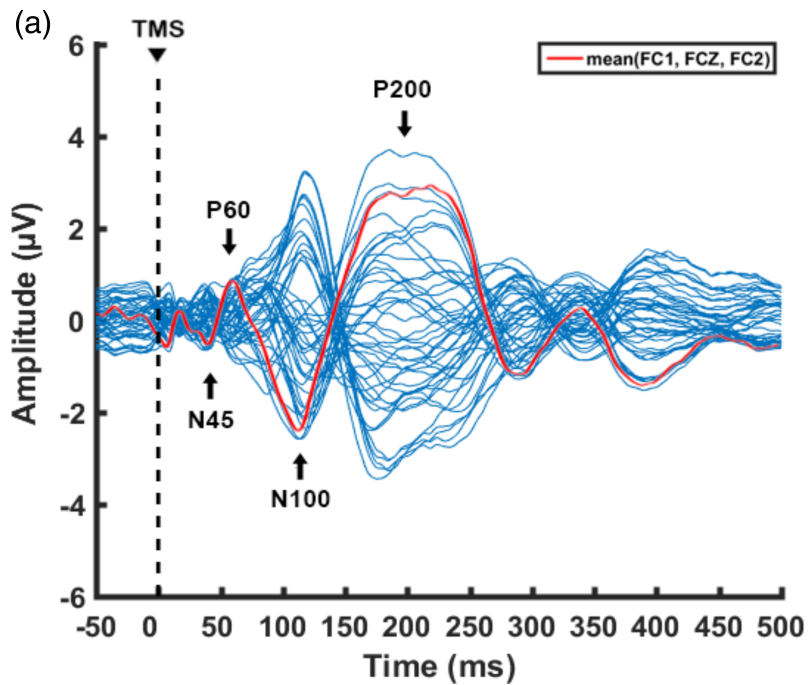

(b) N45
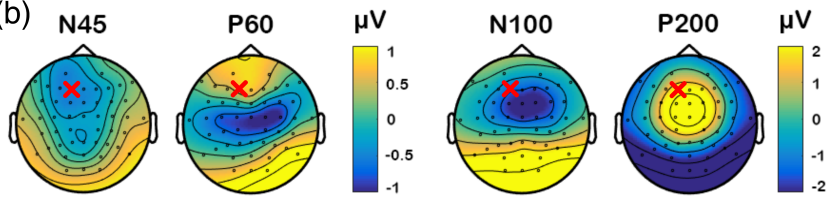

(c)
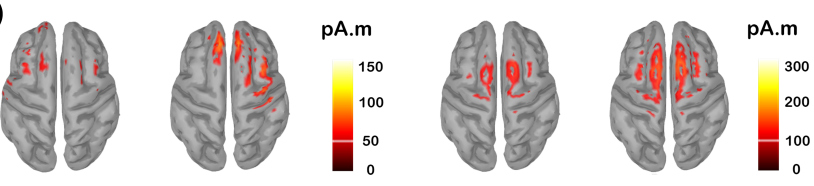

FIGURE 3 Transcranial magnetic stimulation (TMS)-evoked potentials following single-pulse TMS over left prefrontal cortex (F1 electrode) before the application of theta burst stimulation. Data were combined across three different stimulation conditions at baseline. (a) Butterfly plot of all electrodes with peaks of interest indicated in the text. The waveform in red line is formed using the average of three frontocentral electrodes (FC1, FCz, FC2) for graphical representation of prefrontal activity. Topographical distribution of (b) voltage and (c) source activity (minimum norm estimates (MNEs)) at the level of cortex for each peak. " $X$ " on topoplots indicate stimulation site (F1 electrode) [Color figure can be viewed at wileyonlinelibrary.com]

significant differences in iTBS-induced change were found between 30 and $50 \mathrm{~Hz}$ iTBS in any peak at any time point (all $p>.025$ ).

To validate the statistical method used for the comparison of TEPs in this study (nonparametric cluster-based permutation statistics), 3 (iTBS condition) $\times 3$ (time) repeated measures ANOVA was performed using the data extracted from 6 frontal electrodes $(F 1, F z$, $F 2, F C 1, F C z$ and FC2) as described in Section 2.12. The results corroborated the outcomes following cluster-based statistics (Supporting Information Materials, Section S9).

\subsection{Effects of iTBS on the relationship between $\mathrm{P} 60$ and N100}

Previous research has demonstrated relationship between P60 and N100 (Noda, Zomorrodi, Cash, et al., 2017d). We explored whether there was an association in changes in the amplitude of these peaks following iTBS. For this analysis, electrodes were chosen to ensure that changes in P60 and N100 were captured across individuals and comprised F1, Fz, F2, FC1, FCz and FC2 electrodes. While Pearson's 
(a) $30 \mathrm{~Hz}$ iTBS
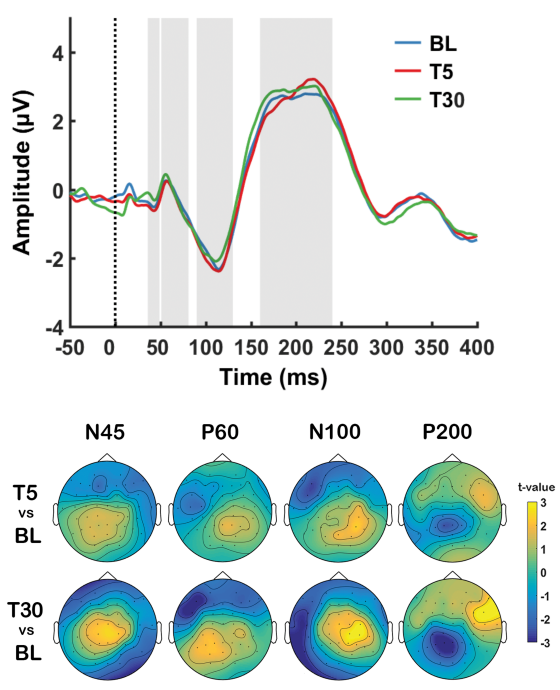

(b) $50 \mathrm{~Hz}$ iTBS
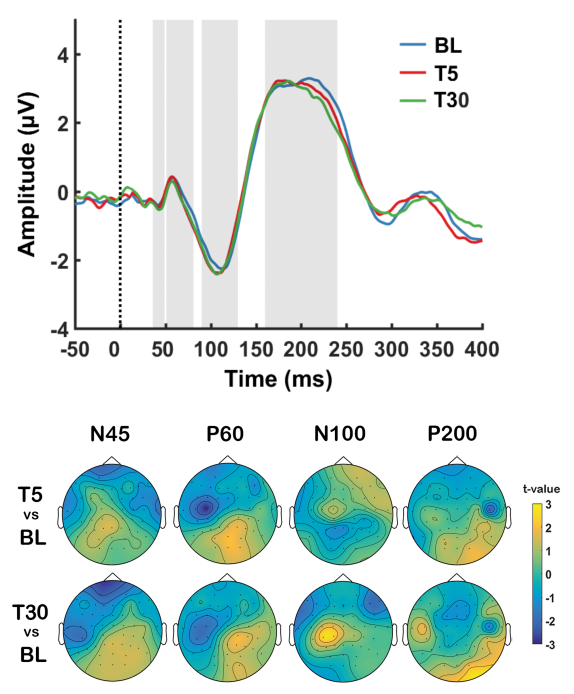

(c) Ind iTBS
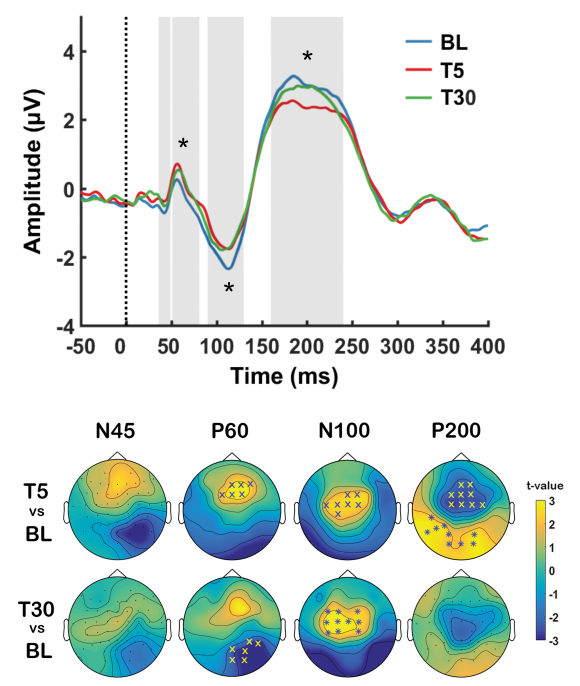

FIGURE 4 Assessment of transcranial magnetic stimulation (TMS)-evoked potentials (TEPs) following different intermittent theta burst stimulation (iTBS) conditions (a: $30 \mathrm{~Hz}$ iTBS, b: $50 \mathrm{~Hz}$ iTBS, c: Ind iTBS). Grand average TEP waveforms at baseline (BL: Blue), 5-min post (T5: Red) and 30-min post (T30: Green) using 3 fronto-central electrodes (FC1, FCz and FC2). Topoplots represent t-values for comparison between time points. Asterisks and "X"s on scalp maps indicate significant sensors between comparisons (cluster-based statistics, ${ }^{*} p<.01,{ }^{\times} p<.025$ ). For visualisation purposes, asterisks have been added to the TEP plots at deflections that were found to be significant in the cluster-based statistics [Color figure can be viewed at wileyonlinelibrary.com]

TABLE 1 Percentage of subjects in which TEPs increased/decreased relative to baseline [Color table can be viewed at wileyonlinelibrary.com]

\begin{tabular}{|c|c|c|c|c|c|c|c|c|c|c|}
\hline & & \multicolumn{3}{|l|}{ P60 } & \multicolumn{3}{|l|}{ N100 } & \multicolumn{3}{|l|}{ P200 } \\
\hline & & $30 \mathrm{~Hz}$ & $50 \mathrm{~Hz}$ & Ind & $30 \mathrm{~Hz}$ & $50 \mathrm{~Hz}$ & Ind & $30 \mathrm{~Hz}$ & $50 \mathrm{~Hz}$ & Ind \\
\hline T5 & $\uparrow$ & $50 \%$ & $45 \%$ & $80 \%$ & $60 \%$ & $45 \%$ & $50 \%$ & $55 \%$ & $50 \%$ & $35 \%$ \\
\hline \multirow[t]{2}{*}{ T30 } & $\uparrow$ & $55 \%$ & $40 \%$ & $75 \%$ & $45 \%$ & $45 \%$ & $30 \%$ & $55 \%$ & $45 \%$ & $45 \%$ \\
\hline & $\downarrow$ & $45 \%$ & $60 \%$ & $25 \%$ & $55 \%$ & $55 \%$ & $70 \%$ & $45 \%$ & $55 \%$ & $55 \%$ \\
\hline
\end{tabular}

Significant changes highlighted in bold (based on cluster-based statistics).

correlations revealed no significant correlations following $30 \mathrm{~Hz}$ iTBS at any time point (T5 $-r=-0.371, p=.108$; T30 $-r=0.298$, $p=.202$ ) (Figure 6a), strong negative correlations were found following $50 \mathrm{~Hz}$ iTBS at both time points (T5 $-r=-0.700, p=.001$; T30 $r=-0.615, p=.004$ ) (Figure 6b) indicating that increased P60 amplitude (more positive) was related to increased N100 amplitude (more negative) following iTBS. Even though this correlation was absent at T5 following Ind iTBS $(r=0.072, p=.763)$, it was present at T30 $(r=-0.710, p=.001)$ (Figure 6c).

\subsection{The effect of different frequency of iTBS on mood rating}

We examined the effects of different iTBS conditions on mood and the relationship between neurophysiological changes and mood changes. The average mood rating before and after each stimulation condition was as follows ( $\mathrm{BL}$ and $\mathrm{T} 60$; mean $\pm S D$; rating out of 100 on VAS, see methods): $30 \mathrm{~Hz}$ iTBS $=77.3 \pm 13.3$ and $78.2 \pm 13.7 ; 50 \mathrm{~Hz}$ iTBS = $76.4 \pm 12.6$ and $77.4 \pm 14.9$; Ind iTBS = $75.6 \pm 13.0$ and $81.4 \pm 11.6$. There were no significant differences between conditions at baseline. One-way repeated measures ANOVA for $\Delta$ mood rating yielded a significant main effect of condition $\left(F_{2,38}=5.495, p=.008, \eta^{2}=0.224\right.$, power $\left.=0.821\right)$. Post-hoc pairwise comparison revealed that $\Delta$ mood was significantly larger following Ind iTBS compared with both $30 \mathrm{~Hz}(p=.024$, Hedges' $g=0.76$, power $=0.897)$ and $50 \mathrm{~Hz}(p=.046$, Hedges' $g=0.73$, power $=$ 0.872 ) iTBS (Figure 7a). No significant difference was found between 30 and $50 \mathrm{~Hz}$ iTBS $(p=1.000$ ) (Details in Supporting Information Materials, Section S6, Table S1).

We next explored which neurophysiological changes, namely $\mathrm{P} 60, \mathrm{~N} 100$ and P200, corresponded to the changes in mood using the combined dataset $(n=60)$. Using the same data from above correlations (average of six fronto-central electrodes), Pearson's correlations revealed a significant positive correlation between $\Delta \operatorname{mood}$ and $\Delta$ P60 at T5 ( $r=0.293, p=.023$ ) (Figure 7b) but not at T30 ( $r=0.055$, $p=.674)$. No significant correlation was found between $\Delta$ mood and $\Delta$ N100 at T5 ( $r=0.203, p=.119)$, but showed a significant positive correlation at T30 ( $r=350, p=.006$ ) (Figure 7c). No significant correlation was found between $\Delta \operatorname{mood}$ and $\Delta \mathrm{P} 200(r=-0.049, p=.713)$ (Figure 7d). A significant positive correlation indicates increased P60 (more positive)/decreased N100 (less negative) corresponds to higher mood rating. 
(a) Ind vs $30 \mathrm{~Hz}$ iTBS
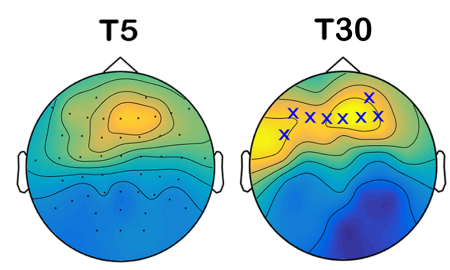

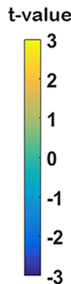

(b) Ind vs $50 \mathrm{~Hz}$ iTBS

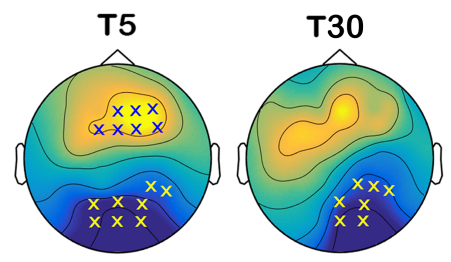

(c)

$30 \mathrm{~Hz}$ iTBS

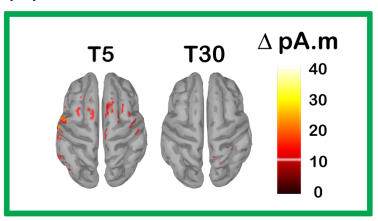

$50 \mathrm{~Hz}$ iTBS

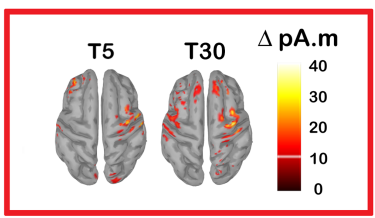

Ind iTBS

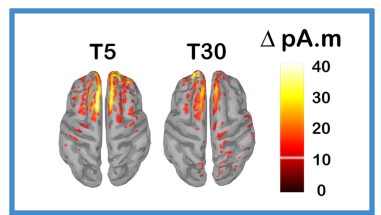

FIGURE 5 Comparison of the change in transcranial magnetic stimulation (TMS)-evoked P60 amplitude between different intermittent theta burst stimulation (iTBS) conditions. Scalp maps represent t-values for comparison of $\Delta$ P60 between (a) Ind iTBS and $30 \mathrm{~Hz}$ iTBS and (b) Ind iTBS and $50 \mathrm{~Hz}$ iTBS. (c) Minimum norm estimates (MNEs) of the source level activity at the cortex for the $\Delta$ P60 peak in different stimulation conditions. "X"s on scalp maps indicate significant sensors between comparisons (cluster-based statistics, $\left.{ }^{\mathrm{X}} p<.025\right)$ [Color figure can be viewed at wileyonlinelibrary.com]

(a) $30 \mathrm{~Hz}$ iTBS

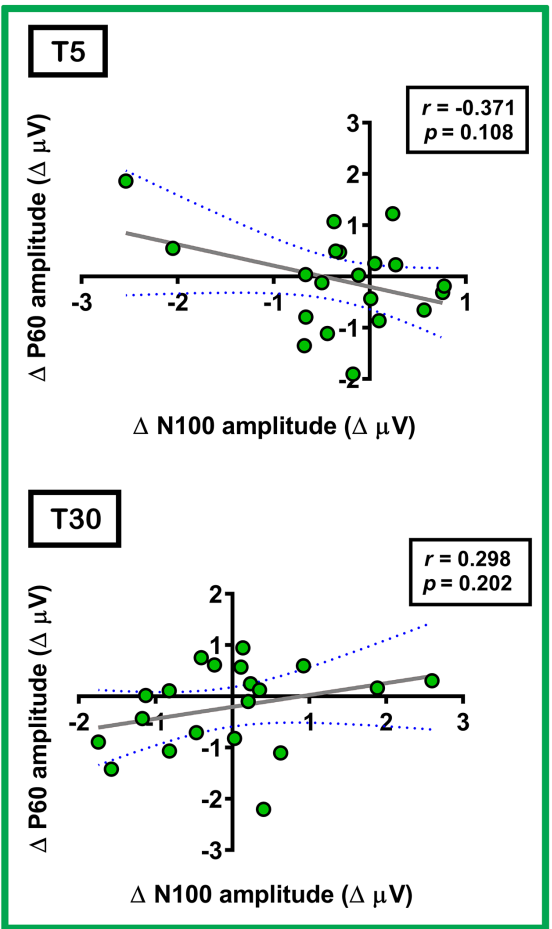

(b) $50 \mathrm{~Hz}$ iTBS

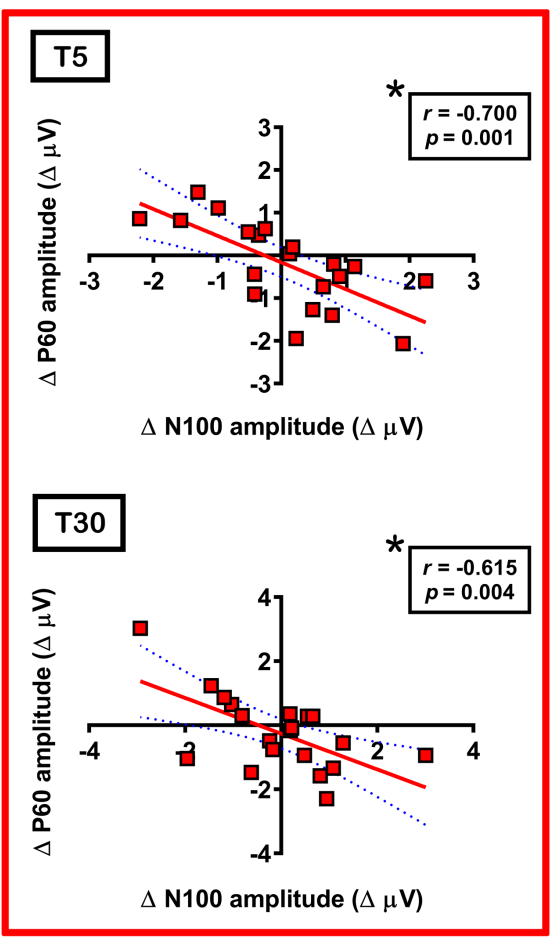

(c) Ind iTBS

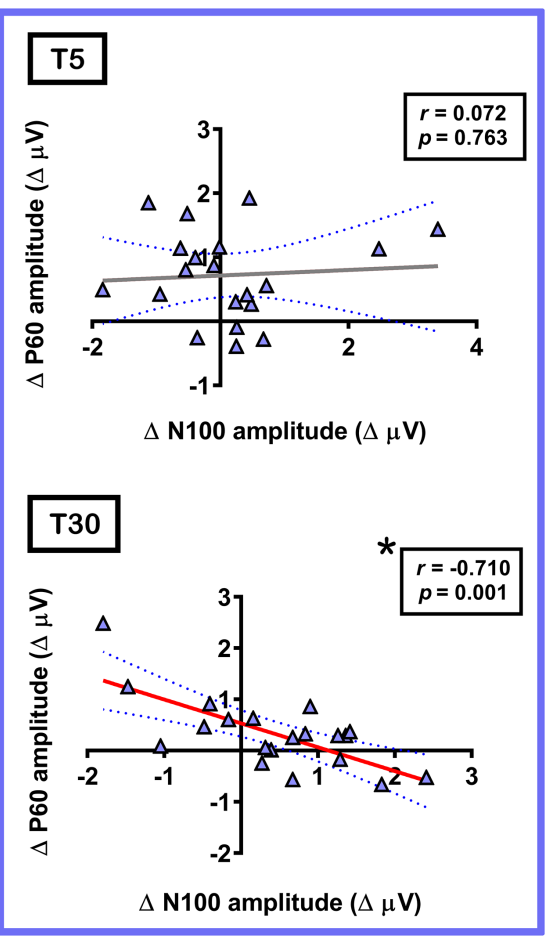

FIGURE 6 Correlations between intermittent theta burst stimulation (iTBS)-induced changes in the amplitude of transcranial magnetic stimulation (TMS)-evoked P60 ( $\Delta$ P60) and TMS-evoked N100 ( $\Delta$ N100) in (a) $30 \mathrm{~Hz}$ iTBS, (b) $50 \mathrm{~Hz}$ iTBS and (c) Ind iTBS. Asterisks indicate significant correlations $(p<.05)$ [Color figure can be viewed at wileyonlinelibrary.com]

\subsection{The effect of different frequency of iTBS on working memory performance}

Order effect analysis was first conducted on the working memory performance at baseline across different stimulation conditions. One-way repeated measures ANOVA resulted in no significant session order effect in either accuracy $\left(F_{2,38}=0.146, p=.865\right)$ or accurate reaction time $\left(F_{2,38}=0.563, p=.574\right)$, confirming the effectiveness of the counter balancing.

Figure 8 illustrates working memory performance assessed via accuracy $\left(d^{\prime}\right)$ and accurate reaction time (in $\mathrm{ms}$ ) in different stimulation conditions over time. Two-way repeated measures ANOVA for $d^{\prime}$ demonstrated a significant interaction between Condition and Time 


\section{○ $30 \mathrm{~Hz}$ iTBS $\square 50 \mathrm{~Hz}$ iTBS $\Delta$ Ind iTBS}
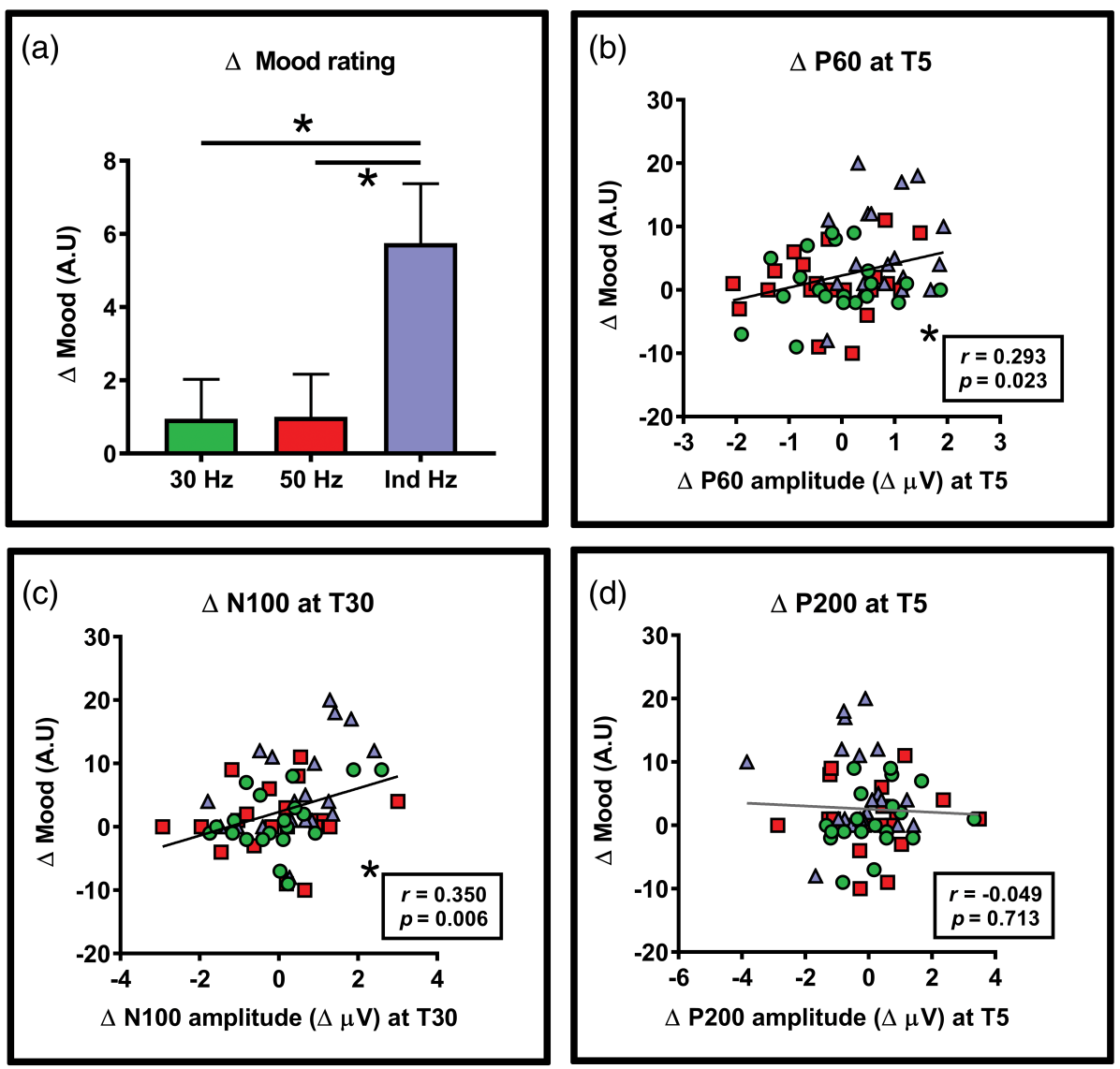

FIGURE 7 Impact of different intermittent theta burst stimulation (iTBS) condition on mood. (a) Significant differences between Ind iTBS and $30 / 50 \mathrm{~Hz}$ iTBS in mood. Correlations between iTBS-induced changes in mood ( $\Delta$ mood) and the amplitude of transcranial magnetic stimulation (TMS)-evoked (b) $\Delta$ P60, (c) $\Delta$ N100 and (d) $\Delta$ P200. Asterisks indicate significant differences/correlations $(p<.05)$ and error bars in (a) indicate standard error of means (SEM) [Color figure can be viewed at wileyonlinelibrary.com]

$\left(F_{4,76}=4.534, p=.002, \eta^{2}=0.193\right.$, power $\left.=0.930\right)$. A series of oneway ANOVAs was performed to further explore the interaction effect. Within condition comparisons resulted no significant main effect of time in the $30 \mathrm{~Hz}$ iTBS condition $\left(F_{2,38}=0.138, p=.871, \eta^{2}=0.007\right.$, power $=0.070$ ), but a significant main effect of time in the $50 \mathrm{~Hz}$ iTBS condition $\left(F_{2,38}=5.905, p=.006, \eta^{2}=0.237\right.$, power $\left.=0.849\right)$, and a significant main effect of time in the Ind iTBS condition $\left(F_{2,38}=7.173, p=.002, \eta^{2}=0.274\right.$, power $\left.=0.913\right)$. Post-hoc pairwise comparisons in $50 \mathrm{~Hz}$ iTBS condition revealed that $d^{\prime}$ was significantly higher at T20 compared with BL ( $p=.028$, Hedges' $g=0.31$, power $=0.261)$ and T45 $(p=.044)$. No significant difference was found between BL and T45 ( $p=.100)$. For Ind iTBS condition, $d^{\prime}$ was significantly higher at T45 compared with both BL ( $p=.029$, Hedges' $g=0.43$, power $=0.447)$ and T20 $(p=.039)$. No significant difference was found between TO and T20 $(p=.100)$ (Details in Supporting Information Materials, Section S7, Table S2).

Between condition comparisons showed no significant main effect at $\mathrm{BL}\left(F_{2,38}=0.407, p=.669, \eta^{2}=0.021\right.$, power $\left.=0.111\right)$, but a significant main effect at T20 $\left(F_{2,38}=4.360, p=.020, \eta^{2}=0.187\right.$, power $=$ $0.721)$ and a trend toward significance at T45 $\left(F_{2,38}=2.688, p=.081\right.$, $\eta^{2}=0.124$, power $=0.501$ ). Post-hoc pairwise comparisons in the main effect at T20 revealed that $d^{\prime}$ was significantly higher following $50 \mathrm{~Hz}$
iTBS compared with Ind iTBS $(p=.015)$, and non-significantly higher compared with $30 \mathrm{~Hz}$ iTBS $(p=.079)$. No significant differences were found between Ind iTBS and $30 \mathrm{~Hz}$ iTBS ( $p=1.000$ ).

To compare the effect sizes between the stimulation conditions, $\Delta$ scores were analysed. One-way ANOVA revealed significant effect of condition at T45 $\left(F_{2,38}=3.463, p=.042, \eta^{2}=0.154\right.$, power $=$ $0.614)$, but not at $\mathrm{T} 20\left(F_{2,38}=2.727, p=.078, \eta^{2}=0.126\right.$, power $=$ 0.507). At T45, Ind iTBS showed higher effect sizes compared with both $30 \mathrm{~Hz}$ (Hedges' $g=0.64$, power $=0.775$ ) and $50 \mathrm{~Hz}$ iTBS (Hedges' $g=0.75$, power $=0.889$ ) (Details in Supporting Information Materials, Section S7, Table S3).

For accurate reaction time, two-way repeated measures ANOVA yielded no significant main effect of condition $\left(F_{2,38}=0.783\right.$, $p=.464, \eta^{2}=0.040$, power $=0.173$ ) and no significant interaction effect $\left(F_{2,38}=0.493, p=.741, \eta^{2}=0.025\right.$, power $\left.=0.161\right)$, but a significant main effect of time $\left(F_{2,38}=4.299, p=.021, \eta^{2}=0.185\right.$, power $=0.714$ ).

Examination of the response to each iTBS condition in $d^{\prime}$ demonstrated large inter-individual variability following $30 \mathrm{~Hz}$ iTBS at both T20 and T45 (Table 2). Reduced variability was seen following $50 \mathrm{~Hz}$ iTBS and Ind iTBS at T20 and T45, respectively. More in-depth exploration of inter-individual variability can be found in Supporting Information Material, Section S8. 

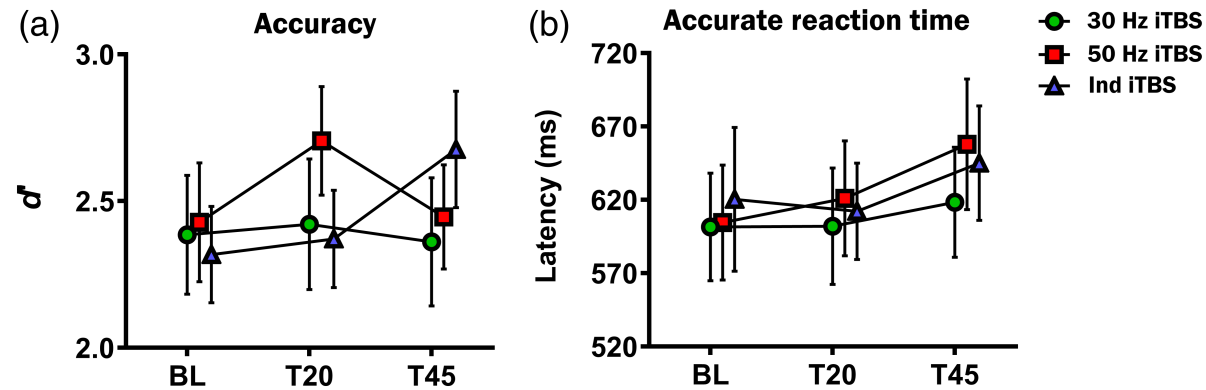

FIGURE 8 Working memory performance (3-back task) assessed via (a) accuracy ( $d^{\prime}$ ) and (b) accurate reaction time (in ms) at BL, T20 and T45 in different intermittent theta burst stimulation condition. Error bars indicate standard error of means (SEM) [Color figure can be viewed at wileyonlinelibrary.com]

We also explored if iTBS-induced changes in $d^{\prime}\left(\Delta d^{\prime}\right)$ had any association with $\Delta \mathrm{P} 60$ and $\Delta \mathrm{N} 100$, but no significant correlations were found $(p>.05)$. We sought to determine if the ratio between $\Delta \mathrm{N} 100$ and $\Delta$ $\mathrm{P} 60(\Delta \mathrm{N} 100 / \Delta \mathrm{P} 60)$ had any influence on the improvement in $d^{\prime}$. This ratio may represent excitation/inhibition balance in the prefrontal cortex (Noda, Zomorrodi, Cash, et al., 2017d) which is important in working memory and network functions (Legon et al., 2016; Rubin, Abbott, \& Sompolinsky, 2017), and balanced regulation is required for prefrontal cortex-dependent behaviours (Fan \& Hu, 2018). The ratio resulted in outliers which were winsorised to fit normal distribution (2 data points each for $30 \mathrm{~Hz}$ iTBS T5 and T30, and Ind iTBS T30). Pearson's correlations revealed no significant correlations in $30 \mathrm{~Hz}$ iTBS condition either at early $(r=0.197, p=.405)$ or late time point $(r=-0.150, p=.541)$ (Figure 9a). In $50 \mathrm{~Hz}$ iTBS condition, a significant correlation was observed at early time point $(r=0.512, p=.021)$, but not at late time point ( $r=0.280, p=.232$ ) (Figure 9b). For Ind iTBS, a significant correlation was found at late time point $(r=0.610, p=.004)$ but not at early time point ( $r=0.364, p=.115$ ) (Figure 9c).

\subsection{Secondary analyses of sham condition and Bayesian inference}

Secondary analyses of sham condition (data collected from a previous study) revealed no significant changes in TEPs, mood or working memory performance over time (Details in Supporting Information Material, Section S10). Effect size comparisons revealed superiority of Ind iTBS over 30 and $50 \mathrm{~Hz}$ iTBS in mood changes (Hedges' g/power: $30 \mathrm{~Hz}$ $\mathrm{iTBS}=0.22 / 0.090 ; 50 \mathrm{~Hz}$ iTBS $=0.22 / 0.090 ;$ Ind iTBS = 0.91/0.674) and 3-back task accuracy at T45 (Hedges' g/power: $30 \mathrm{~Hz}$ iTBS =

TABLE 2 Percentage of subjects in which 3-back task (accuracy and accurate reaction time) increased/decreased relative to baseline [Color table can be viewed at wileyonlinelibrary.com]

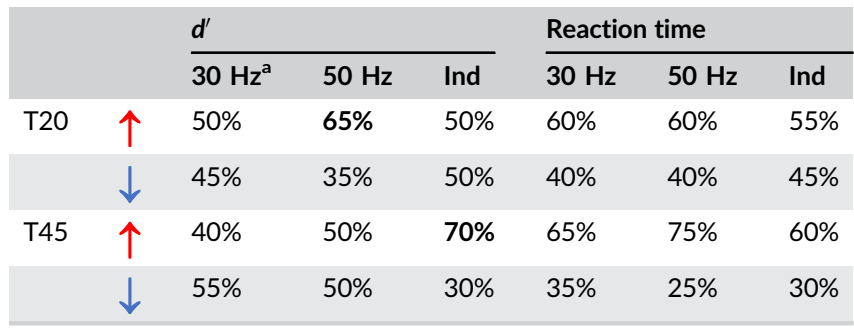

Significant changes highlighted in bold (based on two-way ANOVA).

a Not adding up to $100 \%$ due to 1 subject having no differences in score change $(\Delta=0)$.
0.08/0.055; $\quad 50 \mathrm{~Hz} \quad \mathrm{iTBS}=0.26 / 0.106 ; \quad$ Ind $\quad \mathrm{iTBS}=0.89 / 0.656)$ (Supporting Information Material, Section S10, Tables S4 and S5).

Additional post-hoc Bayesian tests on the accuracy results of the 3-back task confirmed the validity of the current results (Supporting Information Material, Section S11, Table S6).

\section{4 | DISCUSSION}

In this study, we examined the neurophysiological effects of iTBS applied at varying frequency in the prefrontal cortex and the association to LTP-like plasticity. We also investigated whether there was a relationship between iTBS-induced changes in neurophysiology and mood and working memory performance. The data indicate large variability in response to iTBS following both 30 and $50 \mathrm{~Hz}$ iTBS. However, individualised iTBS resulted in more robust changes in neurophysiology and mood compared with standard paradigms. We also demonstrated that working memory may provide a possible behavioural marker of neurophysiological changes following iTBS. The data suggest the frequency of stimulation is an important parameter of iTBS, and a more tailored stimulation protocol may increase the efficacy, and hence could have implications for its therapeutic application.

\subsection{Effect of individualised iTBS on plastic effects in the prefrontal cortex}

Individualised iTBS modulated the amplitude of P60, N100 and P200. The increased P60 amplitude was initially localised around the stimulated area, and later also detected at parieto-occipital sensors. This may represent the propagation of activity across interconnected regions of the cortex over time, a conjecture which is supported by the source localisation (Figure 5c). Such an increase in network level of activity following iTBS has been described during working memory performance (Hoy et al., 2016) and TMS-EEG (Chung, Rogasch, Hoy, Sullivan, et al., 2018b). A similar increase in fronto-parietal P60 has also been observed following a facilitatory neuromodulatory technique, anodal transcranial direct current stimulation (tDCS), in the prefrontal cortex (Hill et al., 2017). A growing body of evidence suggests P60 may provide a marker of cortical excitability. In the motor cortex, P60 amplitude positively correlated with MEP amplitude (Rogasch, Daskalakis, et al., 2013a), and the amplitude was reduced following short-latency afferent inhibition (SAI), an MEP suppression paradigm 
(a) $30 \mathrm{~Hz}$ iTBS

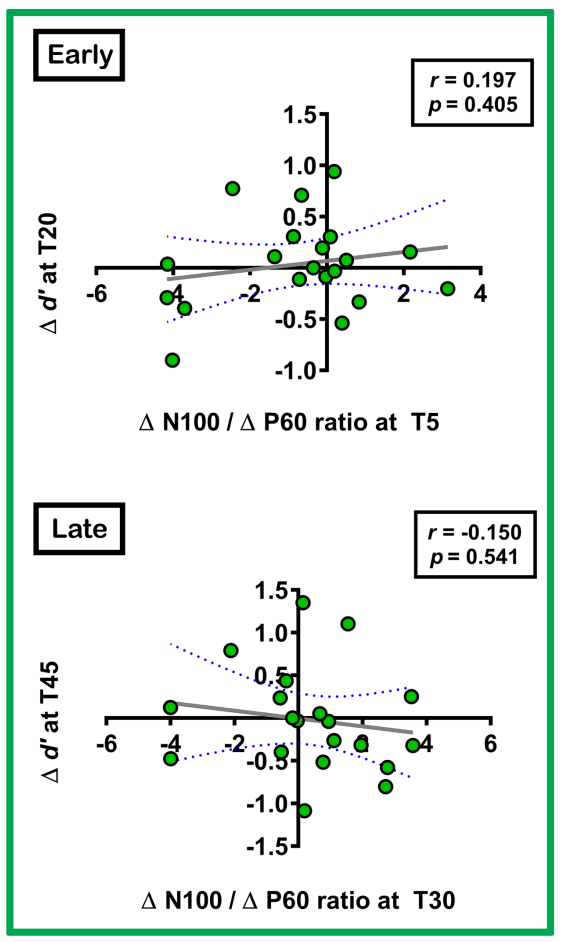

(b) $50 \mathrm{~Hz}$ iTBS

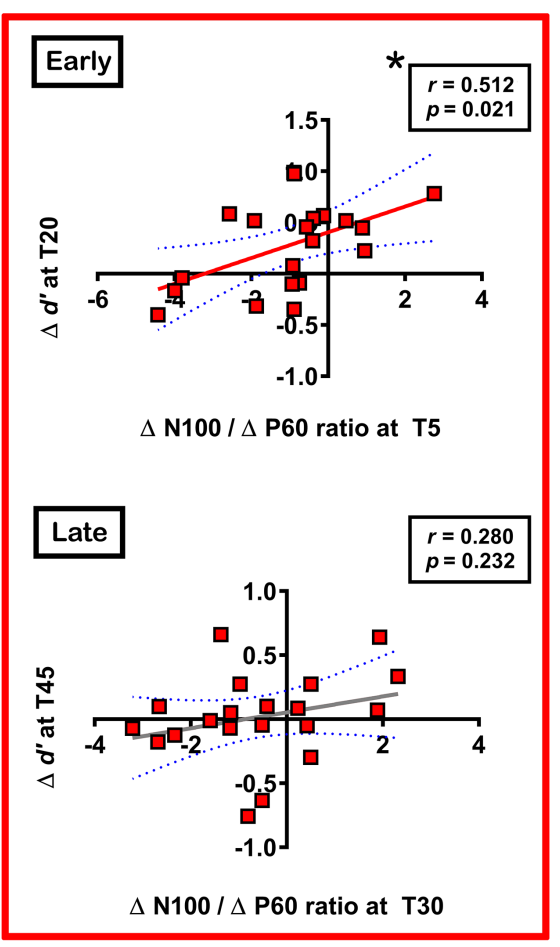

(c) Ind iTBS

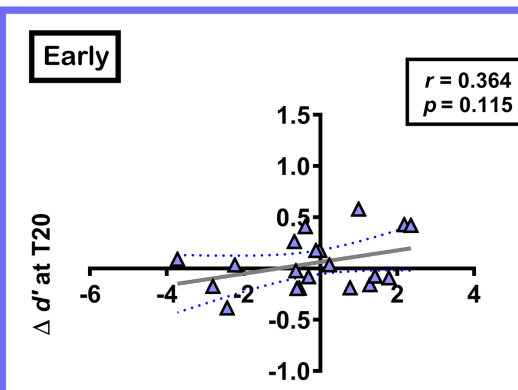

$\Delta \mathrm{N} 100 / \Delta \mathrm{P} 60$ ratio at $\mathrm{T5}$

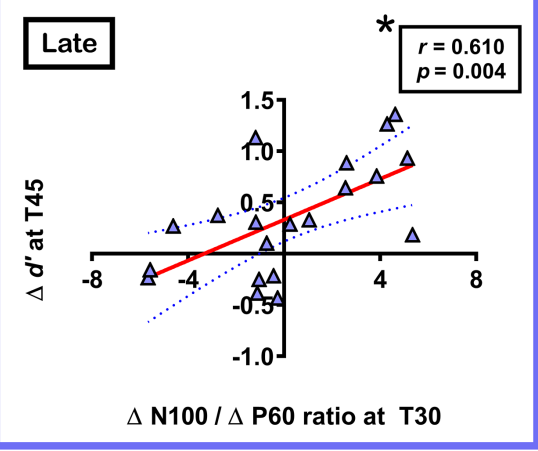

FIGURE 9 Correlations between working memory performance (accuracy; $d^{\prime}$ ) and the ratio of intermittent theta burst stimulation (iTBS)-induced changes in the amplitude of transcranial magnetic stimulation (TMS)-evoked N100 and P60 ( $\Delta$ N100/ $\Delta$ P60) in (a) $30 \mathrm{~Hz}$ iTBS, (b) $50 \mathrm{~Hz}$ iTBS and (c) Ind iTBS. Asterisks indicate significant correlations $(p<.05)$ [Color figure can be viewed at wileyonlinelibrary.com]

(Ferreri et al., 2012). In addition, P60 amplitude was attenuated with short-interval intracortical inhibition (SICl) and increased with intracortical facilitation (ICF) in motor cortex (Cash, Noda, et al., 2017c), concurrent with changes in MEPs. In the prefrontal cortex, SAI resulted in a reduction of this component (Noda, Zomorrodi, Backhouse, et al., 2017c). SICI reduced and ICF increased the amplitude of P60 (Cash, Noda, et al., 2017c), supporting the findings from the motor cortex. Therefore, it is possible that increased P60 amplitude following Ind iTBS reflects enhanced cortical excitability in the prefrontal cortex. The $\Delta$ P60 following Ind iTBS was larger compared with either 30 or $50 \mathrm{~Hz}$ iTBS, particularly around the stimulated region, supported by the cortical activation map.

Individualised stimulation decreased the amplitude of both N100 and P200. While the origin of TMS-evoked P200 is still largely unknown, the physiological property of N100 is more well-defined than other TEPs. The N100 is regarded as the most robust component in TMS-EEG recordings (Noda et al., 2016) with excellent reproducibility (Lioumis, Kicic, Savolainen, Makela, \& Kahkonen, 2009) and signal to noise ratio (Chung et al., 2017). In addition, the N100 deflection is considered to have a high sensitivity to small changes in cortical excitability compared with other TEPs (Nikulin, Kicic, Kahkonen, \& Ilmoniemi, 2003), making it an ideal candidate for tracking neuromodulatory paradigms. Studies have reported N100 to be associated with $\mathrm{GABA}_{B}$-mediated inhibitory mechanisms in both motor (Bonnard et al., 2009; Farzan et al., 2013; Premoli, Rivolta, et al., 2014b; Rogasch, Daskalakis, et al., 2013a) and prefrontal cortex (Chung et al., 2017; Rogasch et al., 2015). The amplitude of N100 increased following SAI both in motor and prefrontal cortex (Noda et al., 2016; Noda,
Zomorrodi, Backhouse, et al., 2017c), but decreased following cerebellar iTBS (Casula, Pellicciari, Ponzo, et al., 2016b), which are in line with the change observed in N100 following Ind iTBS. However, our previous studies showed increased N100 following prefrontal iTBS $(50 \mathrm{~Hz}$ at $5 \mathrm{~Hz}$ ) (Chung et al., 2017; Chung, Rogasch, Hoy, \& Fitzgerald, 2018a; Chung, Rogasch, Hoy, Sullivan, et al., 2018b), and the discrepancy of the outcome is not yet clear. A large body of evidence, mainly in studies with small sample sizes (e.g., $n<15$ ), has suggested that iTBS paradigms increase excitability in the motor cortex (Wischnewski \& Schutter, 2015). However, several studies with large sample sizes $(n>50)$ have found no group-level change in MEPs following $50 \mathrm{~Hz}$ iTBS (Hamada et al., 2013; Lopez-Alonso et al., 2014; Player, Taylor, Alonzo, \& Loo, 2012). Furthermore, a recent meta-analysis demonstrated a degree of publication bias which suggests overestimation of the stimulation effect in the literature (Chung et al., 2016). Similarly, prefrontal iTBS has shown an improvement in working memory in some studies (Hoy et al., 2016; Lowe et al., 2018), however, not in other studies (Chung, Rogasch, Hoy, Sullivan, et al., 2018b; Grossheinrich et al., 2009; Viejo-Sobera et al., 2017). Therefore, the inconsistent results between the current and previous studies may be due to inter-individual variability. Such discrepancy in the after-effects are not only restricted to TBS, but also in other forms of non-invasive brain stimulation (Hill et al., 2017; Muller-Dahlhaus, Orekhov, Liu, \& Ziemann, 2008; Nakamura et al., 2016; Nikolin, Martin, Loo, \& Boonstra, 2018; Wiethoff, Hamada, \& Rothwell, 2014). The variation could be reduced by accounting for the inter-individual neurophysiological differences in parameter design as observed in this study (Ind iTBS) and also in the motor cortex (Cash, Murakami, Chen, Thickbroom, \& Ziemann, 2016). 
By mimicking the original animal study where application of patterned stimulation resembling spike discharge patterns of hippocampal neurons during exploratory behaviours that led to a robust LTP (Larson et al., 1986), it is possible that tailoring the temporal dynamics of pulses to target the individual's disinhibition window (Cash, Ziemann, Murray, \& Thickbroom, 2010; Cash, Ziemann, \& Thickbroom, 2011) yielded a robust LTP-like effect following Ind iTBS. A similar modified approach of stimulation (known as disinhibition stimulation) by individualising the intra and inter-burst frequencies have also shown success in LTP-like plasticity induction in the motor cortex (Cash et al., 2016). While it is difficult to extrapolate from the current results whether Ind cTBS would result in an opposite outcome to Ind iTBS, we anticipate that individualised cTBS might similarly optimise LTD-like effects, given that theta and gamma form the base frequencies for both iTBS and cTBS. LTD-like effects have previously been observed using cTBS (Chung et al., 2017) and pairedassociative stimulation (Casula, Pellicciari, Picazio, et al., 2016a) in the DLPFC, however, a complementary investigation is warranted.

\section{2 | Relationship between iTBS-induced P60 and N100}

On the surface, the observation of no overall effects following both 30 and $50 \mathrm{~Hz}$ iTBS may imply that the two stimulation conditions are indistinguishable. However, the correlation analyses between $\Delta \mathrm{P} 60$ and $\Delta$ N100 demonstrated a close relationship between the peaks following $50 \mathrm{~Hz}$ iTBS which lasted up to $35 \mathrm{~min}$ (see Figure 6b). This pattern was not present in $30 \mathrm{~Hz}$ stimulation (see Figure 6a). In addition, $50 \mathrm{~Hz}$ but not $30 \mathrm{~Hz}$ stimulation produced changes in working memory performance. On the other hand, Ind iTBS temporarily altered this association at T5 with an overall increase in P60 amplitude, which was balanced by larger changes in N100 at T30, indicating a prolonged elevation of the balance between the two peaks (see Figure $6 \mathrm{c}$ ). In the human motor cortex, a pharmacological study demonstrated that $G_{A B A}$ agonists decreased while $G_{A B A}$ agonists increased the amplitude of N100 (Premoli, Castellanos, et al., 2014a). In the instance of the balance between P60 and N100 in this study, a shift in N100 could reflect either an increase in the ratio (reduced $\mathrm{GABA}_{B}$ ) or a shift to maintain the ratio (increased $\mathrm{GABA}_{\mathrm{A}}$ signalling). It is likely to be the latter in this study, and such adaptation of inhibition has also been described in an animal model (Elfant, Pal, Emptage, \& Capogna, 2008; Heiss, Katz, Ganmor, \& Lampl, 2008). These findings are a suggestive reflection of potential metric for LTP (above 0 ) or long-term depression (LTD; below 0) with respect to maintenance of the balance between $\mathrm{P} 60$ and N100, at least following iTBS.

\section{3 | Relationship between neurophysiological changes and the change in mood and working memory performance}

In line with the neurophysiological effects of iTBS, minimal changes were observed in mood rating following both 30 and $50 \mathrm{~Hz}$ iTBS, whereas Ind iTBS resulted in a higher mood rating. Positive correlations were observed only between mood and neurophysiological measures that showed the greatest change (i.e., $\Delta$ P60 at T5, $\Delta$ N100 at T30), suggesting robust physiological changes are required to translate into a behavioural outcome. Another possible explanation is that the mood ratings were re-assessed 60 min after iTBS (T60), at which time the effect of iTBS on the P60 may have been washed out. Alternatively, the early changes in neurophysiology and later changes in mood may be both related to another variable such as a change in connectivity or a delayed onset change in TEPs which was not measured at T60 in this study. The correlations were specific to $\Delta \mathrm{P} 60$ and $\Delta \mathrm{N} 100$, and not with $\Delta \mathrm{P} 200$. Characterisation of P200 is needed to better understand its role, as the changes in this component are often observed following neuromodulation (Casula, Pellicciari, Ponzo, et al., 2016b; Chung et al., 2017; Noda et al., 2016) or in clinical populations (Noda, Barr, Zomorrodi, Cash, Rajji, et al., 2017b).

Studies have demonstrated increased or decreased performance of medium-load (2-back) working memory following left prefrontal iTBS (Hoy et al., 2016) and cTBS (Schicktanz et al., 2015), respectively, using the conventional $50 \mathrm{~Hz}$ protocol (Huang et al., 2005). In the current study, the 3-back task was used and demonstrated to be a potential behavioural marker of neurophysiological changes following iTBS. Investigative studies of different parameters of non-invasive brain stimulation have shown delayed motor cortex physiological effects which could last longer than conventional paradigms (Batsikadze, Moliadze, Paulus, Kuo, \& Nitsche, 2013; Kuo et al., 2013). In addition, working memory performance following iTBS (Chung, Rogasch, Hoy, \& Fitzgerald, 2018a; Hoy et al., 2016) and tDCS (Hill et al., 2017) also demonstrated larger effect sizes at a later time point (30-40 min) compared with early time point (5-20 min). The apparent divergence and a lag between neurophysiological and behavioural effects are not dissimilar to that observed in other recent studies (Hill et al., 2017; Nikolin et al., 2018). However, neural changes often precede behavioural effects (Atienza, Cantero, \& Dominguez-Marin, 2002; Falk, Berkman, Mann, Harrison, \& Lieberman, 2010; Wrase et al., 2007), especially when these effects are mediated by more extensive brain networks. While both $50 \mathrm{~Hz}$ iTBS and Ind iTBS showed improvement in accuracy of the 3-back task compared with baseline, it was evident that Ind iTBS resulted in larger improvement in $d^{\prime}$ scores when the difference from baseline scores were compared (Supporting Information Material S7, Table S3). The effect sizes following Ind iTBS were also larger compared with other conditions, as well as to meta-analyses on working memory using non-invasive brain stimulation including TBS ( $g=0.26$ ) (Brunoni \& Vanderhasselt, 2014; Lowe et al., 2018). The ceiling effect of performance in healthy individuals may have limited more prominent distinction between conditions.

The behavioural outcome of this study was not as direct as anticipated in that the relationship between the neurophysiological measurements ( $\Delta$ TEPs) and working memory performance was not obvious. It is possible that only one hub of the working memory network was modulated, while robust behavioural effects might require modulation of both frontal and parietal hubs of the fronto-parietal working memory network. Alternatively, iTBS was indeed eliciting network-wide effects as has been shown previously (He et al., 2013; Hoy et al., 2016; Rastogi et al., 2017), but the neurophysiological effects were sampled from only one hub (DLPFC) of the frontoparietal working memory network. The reason for $50 \mathrm{~Hz}$ iTBS having an early, short-lasting effect in the accuracy remains unclear. It may be that attentional part of executive functioning was enhanced and 
the effects of stimulation diminished after $30 \mathrm{~min}$. Ind iTBS, on the other hand, resulted in more prolonged network-wide effects, as evidenced by the physiological changes demonstrated using TMS-EEG, and it may have resulted in a delayed behavioural response. In the scenario of bringing about clinical effects, it is often that several weeks of daily brain stimulation sessions are required to generate robust behavioural outcomes (Croarkin et al., 2010; Grall-Bronnec \& Sauvaget, 2014; Perera et al., 2016). Instability, variability or nonconsolidated behavioural changes might account for the differences in time course of the behavioural changes following $50 \mathrm{~Hz}$ and Ind iTBS.

At a mechanistic level, the change in working memory performance may be explained by the physiological changes probed by TMS-EEG. The balanced and dynamic regulation of inhibitory and excitatory activity plays an important role in working memory (Knight, Staines, Swick, \& Chao, 1999; Lim \& Goldman, 2013), which is a critical aspect of animal (Xue, Atallah, \& Scanziani, 2014) and human functional neural circuitry (Cash, Udupa, et al., 2017d; Dehghani et al., 2016). While excitatory plasticity provides a mechanism for learning and memory formation (Froemke, 2015), inhibitory plasticity is essential in maintaining the balance for efficient information processing in cortical networks (Deneve \& Machens, 2016; Vogels, Sprekeler, Zenke, Clopath, \& Gerstner, 2011). The origin of TEPs (P60 and N100) are still largely unknown and no consensus has been reached on what each peak represents. While it is speculative, the correlations between working memory performance and the ratio of P60 and N100 may partially be explained by the well-maintained modulation of potential inhibition (N100) and excitation (P60) (Noda, Zomorrodi, Cash, et al., 2017d). When both $\Delta$ P60 and $\Delta$ N100 showed well-balanced changes ( $50 \mathrm{~Hz}$ iTBS at T5, Ind iTBS at T30), the accuracy increased $(50 \mathrm{~Hz}$ iTBS at T20, Ind iTBS at T45). The physiological changes, namely $\Delta \mathrm{P} 60$ and $\Delta \mathrm{N} 100$, did not correlate with the performance change by themselves, but rather the ratio between the changes in these peaks corresponded with the change in performance. This finding indicates that well-balanced change is more important for working memory than changes in either excitation or inhibition in isolation, as increased excitation alone (as shown in TMS-EEG at T5 in Ind iTBS condition) was not able to enhance the accuracy ( $d^{\prime}$ at T20 in Ind iTBS condition). Such balance is thought to play an important role in cortical processing and working memory (Kirkwood, 2015; Legon et al., 2016; Lim \& Goldman, 2013) and alteration in the balance may lead to cognitive impairment (Cline, 2005; Vogels \& Abbott, 2009). However, although $50 \mathrm{~Hz}$ iTBS showed significant correlations between $\Delta \mathrm{P} 60$ and $\Delta \mathrm{N} 100$ at T30, no sustained improvement was seen in the accuracy of the 3-back task. It is possible that the effect of stimulation was short-lived and may have diminished by T45 when the 3-back task was performed again. In general, the effect of $50 \mathrm{~Hz}$ iTBS in the motor cortex lasts up to $30 \mathrm{~min}$ (Chung et al., 2016). It is unknown if the effects of Ind iTBS would persist longer than T45, and needs to be addressed in the future. However, the elevated mood level at $\mathrm{T} 60$ suggests the effect may have been sustained.

\subsection{Inter-individual variability in the response to conventional iTBS}

A group-level modulation in TEPs was observed following Ind iTBS, but not following 30 or $50 \mathrm{~Hz}$ iTBS. This is in contrast with our previous study in the prefrontal cortex where we observed robust changes following $50 \mathrm{~Hz}$ iTBS, particularly in N100 amplitude (Chung et al., 2017). In addition, there was substantial inter-individual variability in response to iTBS in the current sample (see Table 1). The variability was larger following $30 \mathrm{~Hz}$ iTBS, which showed a large varied response in the direction of change in TEPs (increase or decrease at each time point). A notable amount of opposite directional change was also observed between T5 and T30 (Supporting Information Material, Section S5). The reason for such within-subject variability over time (T5 vs. T30) following $30 \mathrm{~Hz}$ iTBS remains unclear. One possible explanation for this phenomenon is that the effects of $30 \mathrm{~Hz}$ iTBS was short-lived and regulatory homeostatic mechanisms were at play. It was interesting to observe that while $50 \mathrm{~Hz}$ iTBS also resulted in a large inter-individual variability in the change in TEPs, peaks were in the same direction for both T5 and T30 in the majority of subjects. Subtle modifications of the rTMS protocols can influence the effect of stimulation (Cash, Jegatheeswaran, Ni, \& Chen, 2017b; Cash et al., 2016; Ridding \& Ziemann, 2010), and the data suggest frequency of iTBS is an important parameter that can contribute to inter-individual variability. In addition to the stimulation parameters, other factors mediating the outcome include genetics (Antal et al., 2010), attention (Cazzoli, Wurtz, Muri, Hess, \& Nyffeler, 2009), activation of intracortical networks (Hamada et al., 2013), and brain state (Clow et al., 2014; Hinder et al., 2014; Ridding \& Ziemann, 2010).

To our knowledge, $30 \mathrm{~Hz}$ iTBS had not been tested in the prefrontal cortex to date, and our data demonstrate no superiority, and probably inferiority, over $50 \mathrm{~Hz}$ iTBS. It is interesting to note that cerebellar iTBS resulted in the opposite changes in N100 in two separate studies using $50 \mathrm{~Hz}$ (Casula, Pellicciari, Ponzo, et al., 2016b) and $30 \mathrm{~Hz}$ stimulation (Harrington \& Hammond-Tooke, 2015). However, it is unclear whether the outcome was due to varying frequency of stimulation, or a result of inter-individual variability, and more studies are needed to address this discrepancy. In the motor cortex, $30 \mathrm{~Hz}$ cTBS resulted in a larger and more consistent decrease in MEP amplitude than $50 \mathrm{~Hz}$ cTBS (Goldsworthy et al., 2012). Although such a trend was not evident in our data, the differences could be explained by the differences in TBS paradigm (cTBS vs. iTBS), differences in the stimulated region (M1 vs. DLPFC) or the differences in outcome measure (MEPs vs. TEPs). Beyond the motor cortex, Nyffeler et al. targeted the FEF, which is in close proximity to the prefrontal cortex, and found prolonged saccadic delay following $30 \mathrm{~Hz}$ cTBS (Nyffeler et al., 2006b). Saccadic delay has also been reported following $50 \mathrm{~Hz}$ cTBS over the FEF, however, involvement of executive control of saccades were found following prefrontal stimulation (Cameron, Riddle, \& D'Esposito, 2015), suggesting the effect of TBS may be distinct in different brain regions. As there has been no other study using $30 \mathrm{~Hz}$ iTBS in the prefrontal cortex or FEF, future studies are required to confirm and expand these findings. Having no overall effect on neurophysiology, mood and working memory performance following $30 \mathrm{~Hz}$ iTBS in the current study indicates this protocol may not be suitable in the prefrontal cortex. It is critical to note that the carrying frequency was $6 \mathrm{~Hz}$ in $30 \mathrm{~Hz}$ iTBS, and future studies should employ more systematic comparison. We adopted this frequency to be comparable to the direct comparison study in the motor cortex (Goldsworthy et al., 2012), which resulted in a robust decrease in MEPs following $30 \mathrm{~Hz} / 6 \mathrm{~Hz}$ cTBS. 
Although more subjects (70\%; Supporting Information Materials, Section S8, Figure S5) obtained higher $d^{\prime}$ at T45 compared with baseline following Ind iTBS, similar outcome was found at T20 following $50 \mathrm{~Hz}$ iTBS (65\%), and therefore, the decreased behavioural variability in the Ind ITBS condition was not as clear as in the neurophysiological results. The neurophysiological outcome appears to be a more sensitive measure for evaluating the change in brain function following non-invasive brain stimulation than the behavioural effects (Thut $\&$ Pascual-Leone, 2010), as studies have found marked changes in neurophysiology in the absence of significant improvement in the behaviour compared with sham (Chung, Rogasch, Hoy, \& Fitzgerald, 2018a; Hill et al., 2017; Nikolin et al., 2018). Interestingly, neural correlates to behaviour changes were often found in these studies, suggesting the impact of the non-invasive brain stimulation on behaviour may be subtle and the resulting outcome is affected by the variability to a greater extent as was observed in this study. The delayed behavioural response to Ind iTBS makes a direct comparison difficult, especially without the measurement after $45 \mathrm{~min}$ of stimulation. Therefore, it remains inconclusive if Ind iTBS would result in longer-lasting reduction in variability in working memory performance and future research should incorporate extended measurements to supplement more robust behavioural outcomes.

Taken together, these findings suggest that prefrontal iTBS using the conventional parameters of stimulation also suffers from interindividual variability as observed from studies with larger sample sizes in the motor cortex (Hamada et al., 2013; Hinder et al., 2014), and Ind iTBS is able to reduce the variability in neurophysiological change. However, the influence on the variability in behavioural performance remains unclear.

Repetitive TMS of the prefrontal cortex is one therapeutic option for treatment-resistant depression and it is being investigated for use in various mood and cognitive disorders. In order to reduce treatment time, clinics have been exploring the use of short protocols such as TBS (Cash, Dar, et al., 2017a; Desmyter et al., 2016; Duprat et al., 2016; Li et al., 2014), however limited clinical efficacy has also been reported (Prasser et al., 2015). The present data provide the first evidence that using conventional iTBS may not be optimal for neuromodulation, and indicate the need for a more individualised approach of stimulation. A recent meta-analysis investigating the efficacy of TBS in major depression demonstrated a favourable clinical response to treatment, particularly with intervention involving iTBS (Berlim, McGirr, Rodrigues Dos Santos, Tremblay, \& Martins, 2017). Furthermore, a recent clinical trial comparing the antidepressant efficacy between conventional $10 \mathrm{~Hz}$ rTMS and iTBS found similar antidepressant effects in approximately 200 patients in each group (Blumberger et al., 2018), suggesting the possibility of iTBS as a treatment option. However, these studies showed relatively low remission rates following iTBS treatment [18.6\% (Berlim et al., 2017) and 32\% (Blumberger et al., 2018)]. Therefore, the findings of the present study may warrant future clinical trials utilising individualised iTBS as a mechanism to potentially enhance therapeutic outcomes in the treatment of depression. Although we have previously provided evidence for the link between neurophysiology and behavioural effects in healthy individuals (Chung, Rogasch, Hoy, \& Fitzgerald, 2018a; Chung, Rogasch, Hoy, Sullivan, et al., 2018b), the extent to which this will translate to clinical effects remains to be established. Currently, only a small number of studies have examined the effect of TBS on depressed patients using neuroimaging techniques (Li et al., 2018; Pellicciari, Ponzo, Caltagirone, \& Koch, 2017). More studies are needed to elucidate any association between potential clinical efficacy and physiological effects; however, these studies provide a potential link between the modulation of neurophysiology and the clinical improvement following TBS.

It is worth noting, however, that cortical plasticity is disrupted in disorders such as Alzheimer's disease (Battaglia et al., 2007; Koch et al., 2012) and facilitatory paradigms such as iTBS were unable to induce LTP-like effects in this population (Di Lorenzo et al., 2016; Koch et al., 2012, 2016). The variability of the plastic effects were also reduced in this patient group, emphasising the importance and the impact of the intrinsic physiological properties on TBS-induced aftereffects. Furthermore, iTBS may evoke an opposite effect to that of an expected direction (i.e., decrease in MEP amplitude) in patients with spinal cord injury (Fassett et al., 2017), and the resulting outcome may also depend on the cortical area stimulated (Jones et al., 2016). It remains to be determined whether Ind iTBS will elicit desired effects in neurological disorders, and different methods of individualisation may be required for different brain regions.

\section{5 | Limitations}

There are several limitations of the present study. Our study design did not include a primary sham condition. The lack of a control condition limits the potential generalisation of the results. However, the main intention of this study was to compare the effects between the two most commonly used paradigms (which act as active controls) and a novel method of application. While differences between conditions could be determined, non-specific confounds such as time and attention factors were not removed from consideration. Although secondary analyses of data comparing responses to a sham condition demonstrated no significant changes following sham stimulation, it should be noted that volunteers were different from the main analyses, however, were age and gender-ratio matched and had similar years of education. In addition, a control for single-pulse TMS was not included in the study. It is conceivable that single-pulse TMS itself could contribute to the outcomes observed here (Pellicciari, Miniussi, Ferrari, Koch, \& Bortoletto, 2016), however, the experimental design ensured that pre- and post-iTBS measures were consistent across all sessions. Therefore, it is unlikely that single-pulse TMS could have contributed a differential effect that accounted for the observed advantage of individualised iTBS. Furthermore, our previous studies (Chung et al., 2017; Chung, Rogasch, Hoy, \& Fitzgerald, 2018a) indicate no significant change following sham iTBS with a similar experimental design. TMS click sound was masked by white noise but any sound via bone conduction could not be avoided. TMS pulse may induce N100-P200 component in the EEG trace (Ter Braack, de Vos, \& van Putten, 2015), however, it is unlikely that the elicited response is purely auditory-related effect. Any auditory response should be consistent across time, and sham condition did not show any change in these components. In regards to somatosensory input, baseline and post sampling parameters were equivalent across all conditions, including sham (e.g., equal intensity and stimulation site). 
Therefore, the differences between conditions are likely to be driven by cortical plasticity, in accordance with the a priori hypothesis. It should be noted that iTBS-induced changes in TEPs correlated with the changes in mood rating and working memory performance, which suggest TEPs exhibit functional relevance. The selection of iTBS parameters $(50 / 5 \mathrm{~Hz}$ and $30 / 6 \mathrm{~Hz})$ was based on similar parameters used in motor cortex studies. The motor cortex is one region where a measurable output (i.e., MEPs) is obtained following TMS administration. As a result, most studies have used stimulation protocols based on the outcome of motor cortex studies. Future studies would benefit from improving the effect of stimulation tailored to the specific brain region of interest. Due to time constraints, TEPs were not measured at T60 which may have limited more temporally accurate onset of neurophysiological changes to the mood changes. The consistency of the stimulation site could be improved by using MRI-guided neuronavigation, however, this was not feasible in this study. We have taken extra steps to ensure more accurate positioning of the coil. In addition, the TEP waveforms in this study are consistent with other TMSEEG studies in the prefrontal cortex (Chung et al., 2017; Hill et al., 2017; Rogasch et al., 2014), and EEG-guided (Chung et al., 2017; Hill et al., 2017; Rogasch et al., 2014) and MRI-guided (Lioumis et al., 2009) methods have shown comparable results.

\section{5 | CONCLUSIONS}

In conclusion, the current study indicates that the individualised iTBS in the prefrontal cortex is able to exert LTP-like plasticity at a neurophysiological level. The data show a lag and apparent divergence between neurophysiological and behavioural effects. However, neurophysiological changes precede behavioural effects and repeated sessions may be necessary for more consistent and robust behavioural changes. We consider that the present results provide promising and exciting data to suggest the superiority of Ind iTBS at a neurophysiological level and indicate that this may potentially also be the case at a behavioural level.

\section{Disclosures and conflict of interests}

SWC is supported by a Monash Graduate Scholarship. NCR is supported by an NHMRC Early Career Fellowship (1072057). KEH is supported by an NHMRC Career Development Fellowship (1082894). PBF is supported by an NHMRC Practitioner Fellowship (1078567). PBF has received equipment for research from MagVenture $A / S$, Medtronic Ltd, Cervel Neurotech and Brainsway Ltd and funding for research from Neuronetics and Cervel Neurotech. He is on the scientific advisory board for Bionomics Ltd. There are no other conflicts.

\section{ORCID}

Sung Wook Chung (D) https://orcid.org/0000-0001-8022-8037

\section{REFERENCES}

Ahearn, E. P. (1997). The use of visual analog scales in mood disorders: A critical review. Journal of Psychiatric Research, 31, 569-579.
Antal, A., Chaieb, L., Moliadze, V., Monte-Silva, K., Poreisz, C., Thirugnanasambandam, N., ... Paulus, W. (2010). Brain-derived neurotrophic factor (BDNF) gene polymorphisms shape cortical plasticity in humans. Brain Stimulation, 3, 230-237.

Atienza, M., Cantero, J. L., \& Dominguez-Marin, E. (2002). The time course of neural changes underlying auditory perceptual learning. Learning \& Memory, 9, 138-150.

Axmacher, N., Henseler, M. M., Jensen, O., Weinreich, I., Elger, C. E., \& Fell, J. (2010). Cross-frequency coupling supports multi-item working memory in the human hippocampus. Proceedings of the National Academy of Sciences of the United States of America, 107, 3228-3233.

Batsikadze, G., Moliadze, V., Paulus, W., Kuo, M. F., \& Nitsche, M. A. (2013). Partially non-linear stimulation intensity-dependent effects of direct current stimulation on motor cortex excitability in humans. The Journal of Physiology, 591, 1987-2000.

Battaglia, F., Wang, H. Y., Ghilardi, M. F., Gashi, E., Quartarone, A., Friedman, E., \& Nixon, R. A. (2007). Cortical plasticity in Alzheimer's disease in humans and rodents. Biological Psychiatry, 62, 1405-1412.

Berlim, M. T., McGirr, A., Rodrigues Dos Santos, N., Tremblay, S., \& Martins, R. (2017). Efficacy of theta burst stimulation (TBS) for major depression: An exploratory meta-analysis of randomized and sham-controlled trials. Journal of Psychiatric Research, 90, 102-109.

Biel, A. L., \& Friedrich, E. V. C. (2018). Why you should report Bayes factors in your transcranial brain stimulation studies. Frontiers in Psychology, 9, 1125.

Blumberger, D. M., Vila-Rodriguez, F., Thorpe, K. E., Feffer, K., Noda, Y., Giacobbe, P., ... Downar, J. (2018). Effectiveness of theta burst versus high-frequency repetitive transcranial magnetic stimulation in patients with depression (THREE-D): A randomised non-inferiority trial. Lancet, 391, 1683-1692.

Bonansco, C., \& Fuenzalida, M. (2016). Plasticity of hippocampal excitatory-inhibitory balance: Missing the synaptic control in the epileptic brain. Neural Plasticity, 2016, 8607038.

Bonnard, M., Spieser, L., Meziane, H. B., de Graaf, J. B., \& Pailhous, J. (2009). Prior intention can locally tune inhibitory processes in the primary motor cortex: Direct evidence from combined TMS-EEG. The European Journal of Neuroscience, 30, 913-923.

Brownjohn, P. W., Reynolds, J. N., Matheson, N., Fox, J., \& Shemmell, J. B. (2014). The effects of individualized theta burst stimulation on the excitability of the human motor system. Brain Stimulation, 7, 260-268.

Brunoni, A. R., \& Vanderhasselt, M. A. (2014). Working memory improvement with non-invasive brain stimulation of the dorsolateral prefrontal cortex: A systematic review and meta-analysis. Brain and Cognition, 86, 1-9.

Cameron, I. G., Riddle, J. M., \& D'Esposito, M. (2015). Dissociable roles of dorsolateral prefrontal cortex and frontal eye fields during saccadic eye movements. Frontiers in Human Neuroscience, 9, 613.

Cash, R. F. H., Dar, A., Hui, J., De Ruiter, L., Baarbe, J., Fettes, P., ... Chen, R. (2017a). Influence of inter-train interval on the plastic effects of rTMS. Brain Stimulation, 10, 630-636.

Cash, R. F., Jegatheeswaran, G., Ni, Z., \& Chen, R. (2017b). Modulation of the direction and magnitude of Hebbian plasticity in human motor cortex by stimulus intensity and concurrent inhibition. Brain Stimulation, 10, 83-90.

Cash, R. F., Murakami, T., Chen, R., Thickbroom, G. W., \& Ziemann, U. (2016). Augmenting plasticity induction in human motor cortex by disinhibition stimulation. Cerebral Cortex, 26, 58-69.

Cash, R. F., Noda, Y., Zomorrodi, R., Radhu, N., Farzan, F., Rajji, T. K., ... Blumberger, D. M. (2017c). Characterization of glutamatergic and GABAA-mediated neurotransmission in motor and dorsolateral prefrontal cortex using paired-pulse TMS-EEG. Neuropsychopharmacology, 42, 502-511.

Cash, R. F., Udupa, K., Mazzella, F., Daskalakis, Z. J., Wong, A. H., Kennedy, J. L., ... Chen, R. (2017d). Influence of the BDNF Val66Met polymorphism on the balance of excitatory and inhibitory neurotransmission and relationship to plasticity in human cortex. Brain Stimulation, 10, 502.

Cash, R. F., Ziemann, U., Murray, K., \& Thickbroom, G. W. (2010). Late cortical disinhibition in human motor cortex: A triple-pulse transcranial magnetic stimulation study. Journal of Neurophysiology, 103, 511-518. 
Cash, R. F., Ziemann, U., \& Thickbroom, G. W. (2011). Inhibitory and disinhibitory effects on I-wave facilitation in motor cortex. Journal of Neurophysiology, 105, 100-106.

Casula, E. P., Pellicciari, M. C., Picazio, S., Caltagirone, C., \& Koch, G. (2016a). Spike-timing-dependent plasticity in the human dorso-lateral prefrontal cortex. Neurolmage, 143, 204-213.

Casula, E. P., Pellicciari, M. C., Ponzo, V., Stampanoni Bassi, M., Veniero, D., Caltagirone, C., \& Koch, G. (2016b). Cerebellar theta burst stimulation modulates the neural activity of interconnected parietal and motor areas. Scientific Reports, 6, 36191.

Cazzoli, D., Wurtz, P., Muri, R. M., Hess, C. W., \& Nyffeler, T. (2009). Interhemispheric balance of overt attention: A theta burst stimulation study. The European Journal of Neuroscience, 29, 1271-1276.

Chaieb, L., Leszczynski, M., Axmacher, N., Hohne, M., Elger, C. E., \& Fell, J. (2015). Theta-gamma phase-phase coupling during working memory maintenance in the human hippocampus. Cognitive Neuroscience, 6 , 149-157.

Cho, S. S., Ko, J. H., Pellecchia, G., Van Eimeren, T., Cilia, R., \& Strafella, A. P. (2010). Continuous theta burst stimulation of right dorsolateral prefrontal cortex induces changes in impulsivity level. Brain Stimulation, 3, 170-176.

Chung, S. W., Hill, A. T., Rogasch, N. C., Hoy, K. E., \& Fitzgerald, P. B. (2016). Use of theta-burst stimulation in changing excitability of motor cortex: A systematic review and meta-analysis. Neuroscience and Biobehavioral Reviews, 63, 43-64.

Chung, S. W., Lewis, B. P., Rogasch, N. C., Saeki, T., Thomson, R. H., Hoy, K. E., ... Fitzgerald, P. B. (2017). Demonstration of short-term plasticity in the dorsolateral prefrontal cortex with theta burst stimulation: A TMS-EEG study. Clinical Neurophysiology, 128, 1117-1126.

Chung, S. W., Rogasch, N. C., Hoy, K. E., \& Fitzgerald, P. B. (2015). Measuring brain stimulation induced changes in cortical properties using TMS-EEG. Brain Stimulation, 8, 1010-1020.

Chung, S. W., Rogasch, N. C., Hoy, K. E., \& Fitzgerald, P. B. (2018a). The effect of single and repeated prefrontal intermittent theta burst stimulation on cortical reactivity and working memory. Brain Stimulation, 11, 566-574.

Chung, S. W., Rogasch, N. C., Hoy, K. E., Sullivan, C. M., Cash, R. F. H., \& Fitzgerald, P. B. (2018b). Impact of different intensities of intermittent theta burst stimulation on the cortical properties during TMS-EEG and working memory performance. Human Brain Mapping, 39, 783-802.

Cline, H. (2005). Synaptogenesis: A balancing act between excitation and inhibition. Current Biology, 15, R203-R205.

Clow, A., Law, R., Evans, P., Vallence, A. M., Hodyl, N. A., Goldsworthy, M. R., ... Ridding, M. C. (2014). Day differences in the cortisol awakening response predict day differences in synaptic plasticity in the brain. Stress, 17, 219-223.

Cohen, J. (1988). Statistical power analysis for the behavioral sciences (2nd ed.). Hillsdale, NJ: L. Erlbaum Associates.

Conforto, A. B., Z'Graggen, W. J., Kohl, A. S., Rosler, K. M., \& Kaelin-Lang, A. (2004). Impact of coil position and electrophysiological monitoring on determination of motor thresholds to transcranial magnetic stimulation. Clinical Neurophysiology, 115, 812-819.

Croarkin, P. E., Wall, C. A., McClintock, S. M., Kozel, F. A., Husain, M. M., \& Sampson, S. M. (2010). The emerging role for repetitive transcranial magnetic stimulation in optimizing the treatment of adolescent depression. The Journal of ECT, 26, 323-329.

D'Ausilio, A. (2012). Arduino: a low-cost multipurpose lab equipment Behavior Research Methods, 44, 305-313.

Dehghani, N., Peyrache, A., Telenczuk, B., Le Van Quyen, M., Halgren, E., Cash, S. S., ... Destexhe, A. (2016). Dynamic balance of excitation and inhibition in human and monkey neocortex. Scientific Reports, 6, 23176.

Delorme, A., \& Makeig, S. (2004). EEGLAB: An open source toolbox for analysis of single-trial EEG dynamics including independent component analysis. Journal of Neuroscience Methods, 134, 9-21.

Deneve, S., \& Machens, C. K. (2016). Efficient codes and balanced networks. Nature Neuroscience, 19, 375-382.

Desmyter, S., Duprat, R., Baeken, C., Van Autreve, S., Audenaert, K., \& van Heeringen, K. (2016). Accelerated intermittent theta burst stimulation for suicide risk in therapy-resistant depressed patients: A randomized, sham-controlled trial. Frontiers in Human Neuroscience, 10, 480.
Di Lazzaro, V., Pilato, F., Dileone, M., Profice, P., Oliviero, A., Mazzone, P., ... Rothwell, J. C. (2008). The physiological basis of the effects of intermittent theta burst stimulation of the human motor cortex. The Journal of Physiology, 586, 3871-3879.

Di Lorenzo, F., Ponzo, V., Bonni, S., Motta, C., Negrao Serra, P. C., Bozzali, M., ... Koch, G. (2016). Long-term potentiation-like cortical plasticity is disrupted in Alzheimer's disease patients independently from age of onset. Annals of Neurology, 80, 202-210.

Dienes, Z. (2016). How Bayes factors change scientific practice. Journal of Mathematical Psychology, 72, 78-89.

Duprat, R., Desmyter, S., Rudi de, R., van Heeringen, K., Van den Abbeele, D., Tandt, H., ... Baeken, C. (2016). Accelerated intermittent theta burst stimulation treatment in medication-resistant major depression: A fast road to remission? Journal of Affective Disorders, 200, 6-14.

Elfant, D., Pal, B. Z., Emptage, N., \& Capogna, M. (2008). Specific inhibitory synapses shift the balance from feedforward to feedback inhibition of hippocampal CA1 pyramidal cells. The European Journal of Neuroscience, 27, 104-113.

Falk, E. B., Berkman, E. T., Mann, T., Harrison, B., \& Lieberman, M. D. (2010). Predicting persuasion-induced behavior change from the brain. The Journal of Neuroscience, 30, 8421-8424.

Fan, Z., \& Hu, H. (2018). Medial prefrontal cortex excitation/inhibition balance and schizophrenia-like behaviors regulated by thalamic inputs to interneurons. Biological Psychiatry, 83, 630-631.

Farzan, F., Barr, M. S., Hoppenbrouwers, S. S., Fitzgerald, P. B., Chen, R., Pascual-Leone, A., \& Daskalakis, Z. J. (2013). The EEG correlates of the TMS-induced EMG silent period in humans. Neurolmage, 83, 120-134.

Fassett, H. J., Turco, C. V., El-Sayes, J., Lulic, T., Baker, S., Richardson, B., \& Nelson, A. J. (2017). Transcranial magnetic stimulation with intermittent theta burst stimulation alters corticospinal output in patients with chronic incomplete spinal cord injury. Frontiers in Neurology, 8, 380.

Faul, F., Erdfelder, E., Buchner, A., \& Lang, A. G. (2009). Statistical power analyses using $\mathrm{G}^{*}$ power 3.1: Tests for correlation and regression analyses. Behavior Research Methods, 41, 1149-1160.

Faul, F., Erdfelder, E., Lang, A. G., \& Buchner, A. (2007). G*power 3: A flexible statistical power analysis program for the social, behavioral, and biomedical sciences. Behavior Research Methods, 39, 175-191.

Ferreri, F., Ponzo, D., Hukkanen, T., Mervaala, E., Kononen, M., Pasqualetti, P., ... Maatta, S. (2012). Human brain cortical correlates of short-latency afferent inhibition: A combined EEG-TMS study. Journal of Neurophysiology, 108, 314-323.

Friese, U., Koster, M., Hassler, U., Martens, U., Trujillo-Barreto, N., \& Gruber, T. (2013). Successful memory encoding is associated with increased cross-frequency coupling between frontal theta and posterior gamma oscillations in human scalp-recorded EEG. Neurolmage, 66, 642-647.

Froemke, R. C. (2015). Plasticity of cortical excitatory-inhibitory balance. Annual Review of Neuroscience, 38, 195-219.

Goldsworthy, M. R., Pitcher, J. B., \& Ridding, M. C. (2012). A comparison of two different continuous theta burst stimulation paradigms applied to the human primary motor cortex. Clinical Neurophysiology, 123 2256-2263.

Grall-Bronnec, M., \& Sauvaget, A. (2014). The use of repetitive transcranial magnetic stimulation for modulating craving and addictive behaviours: A critical literature review of efficacy, technical and methodological considerations. Neuroscience and Biobehavioral Reviews, 47, 592-613.

Gramfort, A., Papadopoulo, T., Olivi, E., \& Clerc, M. (2010). OpenMEEG: Opensource software for quasistatic bioelectromagnetics. Biomedical Engineering Online, 9, 45.

Grossheinrich, N., Rau, A., Pogarell, O., Hennig-Fast, K., Reinl, M., Karch, S., ... Padberg, F. (2009). Theta burst stimulation of the prefrontal cortex: Safety and impact on cognition, mood, and resting electroencephalogram. Biological Psychiatry, 65, 778-784.

Haatveit, B. C., Sundet, K., Hugdahl, K., Ueland, T., Melle, I., \& Andreassen, O. A. (2010). The validity of $d$ prime as a working memory index: Results from the "Bergen n-back task". The Journal of Clinical and Experimental Neuropsychology, 32, 871-880.

Hamada, M., Murase, N., Hasan, A., Balaratnam, M., \& Rothwell, J. C. (2013). The role of interneuron networks in driving human motor cortical plasticity. Cerebral Cortex, 23, 1593-1605. 
Harrington, A., \& Hammond-Tooke, G. D. (2015). Theta burst stimulation of the cerebellum modifies the TMS-evoked N100 potential, a marker of GABA inhibition. PLoS One, 10, e0141284.

He, X., Lan, Y., Xu, G., Mao, Y., Chen, Z., Huang, D., \& Pei, Z. (2013). Frontoparietal regions may become hypoactive after intermittent theta burst stimulation over the contralateral homologous cortex in humans. Journal of Neurophysiology, 110, 2849-2856.

Heiss, J. E., Katz, Y., Ganmor, E., \& Lampl, I. (2008). Shift in the balance between excitation and inhibition during sensory adaptation of S1 neurons. The Journal of Neuroscience, 28, 13320-13330.

Hill, A. T., Rogasch, N. C., Fitzgerald, P. B., \& Hoy, K. E. (2017). Effects of prefrontal bipolar and high-definition transcranial direct current stimulation on cortical reactivity and working memory in healthy adults. Neurolmage, 152, 142-157.

Hinder, M. R., Goss, E. L., Fujiyama, H., Canty, A. J., Garry, M. I., Rodger, J., \& Summers, J. J. (2014). Inter- and intra-individual variability following intermittent theta burst stimulation: Implications for rehabilitation and recovery. Brain Stimulation, 7, 365-371.

Hoy, K. E., Bailey, N., Michael, M., Fitzgibbon, B., Rogasch, N. C., Saeki, T., \& Fitzgerald, P. B. (2016). Enhancement of working memory and task-related oscillatory activity following intermittent theta burst stimulation in healthy controls. Cerebral Cortex, 26, 4563-4573.

Huang, Y. Z., Edwards, M. J., Rounis, E., Bhatia, K. P., \& Rothwell, J. C. (2005). Theta burst stimulation of the human motor cortex. Neuron, 45 201-206.

Jacobs, J. (2014). Hippocampal theta oscillations are slower in humans than in rodents: Implications for models of spatial navigation and memory. Philosophical Transactions of the Royal Society of London. Series B, Biological Sciences, 369, 20130304.

Jones, C. B., Lulic, T., Bailey, A. Z., Mackenzie, T. N., Mi, Y. Q., Tommerdahl, M., \& Nelson, A. J. (2016). Metaplasticity in human primary somatosensory cortex: Effects on physiology and tactile perception. Journal of Neurophysiology, 115, 2681-2691.

Kirkwood, A. (2015). Balancing excitation and inhibition. Neuron, 86, 348-350.

Klimesch, W., Doppelmayr, M., Russegger, H., \& Pachinger, T. (1996). Theta band power in the human scalp EEG and the encoding of new information. Neuroreport, 7, 1235-1240.

Knight, R. T., Staines, W. R., Swick, D., \& Chao, L. L. (1999). Prefrontal cortex regulates inhibition and excitation in distributed neural networks. Acta Psychologica, 101, 159-178.

Koch, G., Di Lorenzo, F., Bonni, S., Ponzo, V., Caltagirone, C., \& Martorana, A. (2012). Impaired LTP- but not LTD-like cortical plasticity in Alzheimer's disease patients. Journal of Alzheimer's Disease, 31, 593-599.

Koch, G., Di Lorenzo, F., Del Olmo, M. F., Bonni, S., Ponzo, V., Caltagirone, C., ... Martorana, A. (2016). Reversal of LTP-like cortical plasticity in Alzheimer's disease patients with tau-related faster clinical progression. Journal of Alzheimer's Disease, 50, 605-616.

Koessler, L., Maillard, L., Benhadid, A., Vignal, J. P., Felblinger, J., Vespignani, H., \& Braun, M. (2009). Automated cortical projection of EEG sensors: Anatomical correlation via the international $10-10$ system. Neurolmage, 46, 64-72.

Koster, M., Friese, U., Schone, B., Trujillo-Barreto, N., \& Gruber, T. (2014). Theta-gamma coupling during episodic retrieval in the human EEG. Brain Research, 1577, 57-68.

Kuo, H. I., Bikson, M., Datta, A., Minhas, P., Paulus, W., Kuo, M. F., \& Nitsche, M. A. (2013). Comparing cortical plasticity induced by conventional and high-definition $4 \times 1$ ring tDCS: A neurophysiological study. Brain Stimulation, 6, 644-648.

Larson, J., \& Munkacsy, E. (2015). Theta-burst LTP. Brain Research, 1621, $38-50$.

Larson, J., Wong, D., \& Lynch, G. (1986). Patterned stimulation at the theta frequency is optimal for the induction of hippocampal long-term potentiation. Brain Research, 368, 347-350.

Le-Niculescu, H., Kurian, S. M., Yehyawi, N., Dike, C., Patel, S. D., Edenberg, H. J., ... Niculescu, A. B. (2009). Identifying blood biomarkers for mood disorders using convergent functional genomics. Molecular Psychiatry, 14, 156-174.
Lee, T. G., \& D'Esposito, M. (2012). The dynamic nature of top-down signals originating from prefrontal cortex: A combined fMRI-TMS study. The Journal of Neuroscience, 32, 15458-15466.

Legon, W., Punzell, S., Dowlati, E., Adams, S. E., Stiles, A. B., \& Moran, R. J. (2016). Altered prefrontal excitation/inhibition balance and prefrontal output: Markers of aging in human memory networks. Cerebral Cortex, 26, 4315-4326.

Li, C. T., Chen, M. H., Juan, C. H., Huang, H. H., Chen, L. F., Hsieh, J. C., ... $\mathrm{Su}, \mathrm{T}$. P. (2014). Efficacy of prefrontal theta-burst stimulation in refractory depression: A randomized sham-controlled study. Brain, 137, 2088-2098.

Li, C. T., Chen, M. H., Juan, C. H., Liu, R. S., Lin, W. C., Bai, Y. M., \& Su, T. P. (2018). Effects of prefrontal theta-burst stimulation on brain function in treatment-resistant depression: A randomized sham-controlled neuroimaging study. Brain Stimulation, 11, 1054-1062.

Lim, S., \& Goldman, M. S. (2013). Balanced cortical microcircuitry for maintaining information in working memory. Nature Neuroscience, 16, 1306-1314.

Lin, F. H., Belliveau, J. W., Dale, A. M., \& Hamalainen, M. S. (2006). Distributed current estimates using cortical orientation constraints. Human Brain Mapping, 27, 1-13.

Lioumis, P., Kicic, D., Savolainen, P., Makela, J. P., \& Kahkonen, S. (2009). Reproducibility of TMS-evoked EEG responses. Human Brain Mapping, 30, 1387-1396.

Lisman, J. (2010). Working memory: The importance of theta and gamma oscillations. Current Biology, 20, R490-R492.

Lisman, J. (2012). Excitation, inhibition, local oscillations, or large-scale loops: What causes the symptoms of schizophrenia? Current Opinion in Neurobiology, 22, 537-544.

Lisman, J. E., \& Jensen, O. (2013). The theta-gamma neural code. Neuron, 77, 1002-1016.

Lopez-Alonso, V., Cheeran, B., Rio-Rodriguez, D., \& Fernandez-Del-Olmo, M. (2014). Inter-individual variability in response to non-invasive brain stimulation paradigms. Brain Stimulation, 7, 372-380.

Lowe, C. J., Manocchio, F., Safati, A. B., \& Hall, P. A. (2018). The effects of theta burst stimulation (TBS) targeting the prefrontal cortex on executive functioning: A systematic review and meta-analysis. Neuropsychologia, 111, 344-359.

Maris, E., \& Oostenveld, R. (2007). Nonparametric statistical testing of EEG- and MEG-data. Journal of Neuroscience Methods, 164, 177-190.

Meunier, C. N., Chameau, P., \& Fossier, P. M. (2017). Modulation of synaptic plasticity in the cortex needs to understand all the players. Frontiers in Synaptic Neuroscience, 9, 2.

Moreau, A. W., Amar, M., Callebert, J., \& Fossier, P. (2013). Serotonergic modulation of LTP at excitatory and inhibitory synapses in the developing rat visual cortex. Neuroscience, 238, 148-158.

Muller-Dahlhaus, J. F., Orekhov, Y., Liu, Y., \& Ziemann, U. (2008). Interindividual variability and age-dependency of motor cortical plasticity induced by paired associative stimulation. Experimental Brain Research, 187, 467-475.

Nakamura, K., Groiss, S. J., Hamada, M., Enomoto, H., Kadowaki, S., Abe, M., ... Ugawa, Y. (2016). Variability in response to Quadripulse stimulation of the motor cortex. Brain Stimulation, 9, 859-866.

Nikolin, S., Martin, D., Loo, C. K., \& Boonstra, T. W. (2018). Effects of TDCS dosage on working memory in healthy participants. Brain Stimulation, 11, 518-527.

Nikouline, V., Ruohonen, J., \& Ilmoniemi, R. J. (1999). The role of the coil click in TMS assessed with simultaneous EEG. Clinical Neurophysiology, 110, 1325-1328.

Nikulin, V. V., Kicic, D., Kahkonen, S., \& IImoniemi, R. J. (2003). Modulation of electroencephalographic responses to transcranial magnetic stimulation: Evidence for changes in cortical excitability related to movement. The European Journal of Neuroscience, 18, 1206-1212.

Noda, Y., Barr, M. S., Zomorrodi, R., Cash, R. F. H., Farzan, F., Rajji, T. K., ... Blumberger, D. M. (2017a). Evaluation of short interval cortical inhibition and intracortical facilitation from the dorsolateral prefrontal cortex in patients with schizophrenia. Scientific Reports, 7, 17106.

Noda, Y., Barr, M. S., Zomorrodi, R., Cash, R. F., Rajji, T. K., Farzan, F., ... Blumberger, D. M. (2017b). Reduced short-latency afferent inhibition in prefrontal but not motor cortex and its association with executive 
function in schizophrenia: A combined TMS-EEG study. Schizophrenia Bulletin, 43, S141.

Noda, Y., Cash, R. F., Zomorrodi, R., Dominguez, L. G., Farzan, F., Rajji, T. K., ... Blumberger, D. M. (2016). A combined TMS-EEG study of short-latency afferent inhibition in the motor and dorsolateral prefrontal cortex. Journal of Neurophysiology, 116, 938-948.

Noda, Y., Zomorrodi, R., Backhouse, F., Cash, R. F. H., Barr, M. S., Rajji, T. K., ... Blumberger, D. M. (2017c). Reduced prefrontal short-latency afferent inhibition in older adults and its relation to executive function: A TMS-EEG study. Frontiers in Aging Neuroscience, 9, 119.

Noda, Y., Zomorrodi, R., Cash, R. F., Barr, M. S., Farzan, F., Rajji, T. K., ... Blumberger, D. M. (2017d). Characterization of the influence of age on GABAA and glutamatergic mediated functions in the dorsolateral prefrontal cortex using paired-pulse TMS-EEG. Aging (Albany NY), 9, 556-572.

Nyffeler, T., Wurtz, P., Luscher, H. R., Hess, C. W., Senn, W., Pflugshaupt, T., ... Muri, R. M. (2006a). Extending lifetime of plastic changes in the human brain. The European Journal of Neuroscience, 24, 2961-2966.

Nyffeler, T., Wurtz, P., Luscher, H. R., Hess, C. W., Senn, W., Pflugshaupt, T., ... Muri, R. M. (2006b). Repetitive TMS over the human oculomotor cortex: Comparison of $1-\mathrm{Hz}$ and theta burst stimulation. Neuroscience Letters, 409, 57-60.

Oostenveld, R., Fries, P., Maris, E., \& Schoffelen, J. M. (2011). FieldTrip: Open source software for advanced analysis of MEG, EEG, and invasive electrophysiological data. Computational Intelligence and Neuroscience, 2011, 156869.

Opie, G. M., Rogasch, N. C., Goldsworthy, M. R., Ridding, M. C., \& Semmler, J. G. (2017). Investigating TMS-EEG indices of long-interval Intracortical inhibition at different Interstimulus intervals. Brain Stimulation, 10, 65-74.

Park, J. Y., Jhung, K., Lee, J., \& An, S. K. (2013). Theta-gamma coupling during a working memory task as compared to a simple vigilance task. Neuroscience Letters, 532, 39-43.

Pellicciari, M. C., Miniussi, C., Ferrari, C., Koch, G., \& Bortoletto, M. (2016). Ongoing cumulative effects of single TMS pulses on corticospinal excitability: An intra- and inter-block investigation. Clinical Neurophysiology, 127, 621-628.

Pellicciari, M. C., Ponzo, V., Caltagirone, C., \& Koch, G. (2017). Restored asymmetry of prefrontal cortical oscillatory activity after bilateral theta burst stimulation treatment in a patient with major depressive disorder: A TMS-EEG study. Brain Stimulation, 10, 147-149.

Penny, W. D., Duzel, E., Miller, K. J., \& Ojemann, J. G. (2008). Testing for nested oscillation. Journal of Neuroscience Methods, 174, 50-61.

Perera, T., George, M. S., Grammer, G., Janicak, P. G., Pascual-Leone, A., \& Wirecki, T. S. (2016). The clinical TMS Society consensus review and treatment recommendations for TMS therapy for major depressive disorder. Brain Stimulation, 9, 336-346.

Player, M. J., Taylor, J. L., Alonzo, A., \& Loo, C. K. (2012). Paired associative stimulation increases motor cortex excitability more effectively than theta-burst stimulation. Clinical Neurophysiology, 123, 2220-2226.

Prasser, J., Schecklmann, M., Poeppl, T. B., Frank, E., Kreuzer, P. M., Hajak, G., ... Langguth, B. (2015). Bilateral prefrontal rTMS and theta burst TMS as an add-on treatment for depression: A randomized placebo controlled trial. The World Journal of Biological Psychiatry, 16, 57-65.

Premoli, I., Bergmann, T. O., Fecchio, M., Rosanova, M., Biondi, A., Belardinelli, P., \& Ziemann, U. (2017). The impact of GABAergic drugs on TMS-induced brain oscillations in human motor cortex. Neurolmage, 163, 1-12.

Premoli, I., Castellanos, N., Rivolta, D., Belardinelli, P., Bajo, R., Zipser, C., ... Ziemann, U. (2014a). TMS-EEG signatures of GABAergic neurotransmission in the human cortex. The Journal of Neuroscience, 34, 5603-5612.

Premoli, I., Rivolta, D., Espenhahn, S., Castellanos, N., Belardinelli, P., Ziemann, U., \& Muller-Dahlhaus, F. (2014b). Characterization of GABAB-receptor mediated neurotransmission in the human cortex by paired-pulse TMS-EEG. Neurolmage, 103, 152-162.

Rastogi, A., Cash, R., Dunlop, K., Vesia, M., Kucyi, A., Ghahremani, A., ... Chen, R. (2017). Modulation of cognitive cerebello-cerebral functional connectivity by lateral cerebellar continuous theta burst stimulation. Neurolmage, 158, 48-57.

Ridding, M. C., \& Ziemann, U. (2010). Determinants of the induction of cortical plasticity by non-invasive brain stimulation in healthy subjects. The Journal of Physiology, 588, 2291-2304.

Robinson, O. J., Cools, R., Crockett, M. J., \& Sahakian, B. J. (2010). Mood state moderates the role of serotonin in cognitive biases. Journal of Psychopharmacology, 24, 573-583.

Robinson, O. J., \& Sahakian, B. J. (2009). A double dissociation in the roles of serotonin and mood in healthy subjects. Biological Psychiatry, 65, 89-92.

Rogasch, N. C., Daskalakis, Z. J., \& Fitzgerald, P. B. (2013a). Mechanisms underlying long-interval cortical inhibition in the human motor cortex: A TMS-EEG study. Journal of Neurophysiology, 109, 89-98.

Rogasch, N. C., Daskalakis, Z. J., \& Fitzgerald, P. B. (2015). Cortical inhibition of distinct mechanisms in the dorsolateral prefrontal cortex is related to working memory performance: A TMS-EEG study. Cortex, 64, 68-77.

Rogasch, N. C., Sullivan, C., Thomson, R. H., Rose, N. S., Bailey, N. W., Fitzgerald, P. B., ... Hernandez-Pavon, J. C. (2017). Analysing concurrent transcranial magnetic stimulation and electroencephalographic data: A review and introduction to the open-source TESA software. Neurolmage, 147, 934-951.

Rogasch, N. C., Thomson, R. H., Daskalakis, Z. J., \& Fitzgerald, P. B. (2013b). Short-latency artifacts associated with concurrent TMS-EEG. Brain Stimulation, 6, 868-876.

Rogasch, N. C., Thomson, R. H., Farzan, F., Fitzgibbon, B. M., Bailey, N. W., Hernandez-Pavon, J. C., ... Fitzgerald, P. B. (2014). Removing artefacts from TMS-EEG recordings using independent component analysis: Importance for assessing prefrontal and motor cortex network properties. Neurolmage, 101, 425-439.

Rubin, R., Abbott, L. F., \& Sompolinsky, H. (2017). Balanced excitation and inhibition are required for high-capacity, noise-robust neuronal selectivity. Proceedings of the National Academy of Sciences of the United States of America, 114, E9366-E9375.

Schack, B., Vath, N., Petsche, H., Geissler, H. G., \& Moller, E. (2002). Phase-coupling of theta-gamma EEG rhythms during short-term memory processing. International Journal of Psychophysiology, 44, 143-163.

Schicktanz, N., Fastenrath, M., Milnik, A., Spalek, K., Auschra, B., Nyffeler, T., ... Schwegler, K. (2015). Continuous theta burst stimulation over the left dorsolateral prefrontal cortex decreases medium load working memory performance in healthy humans. PLoS One, 10, e0120640.

Schubert, T. W., D'Ausilio, A., \& Canto, R. (2013). Using Arduino microcontroller boards to measure response latencies. Behavior Research Methods, 45, 1332-1346.

Schultz, B. G., \& van Vugt, F. T. (2016). Tap Arduino: An Arduino microcontroller for low-latency auditory feedback in sensorimotor synchronization experiments. Behavior Research Methods, 48, 1591-1607.

Sheehan, D. V., Lecrubier, Y., Sheehan, K. ., Amorim, P., Janavs, J., Weiller, E., Hergueta, T., Baker, R., Dunbar, G. C., 1998. The mini-international neuropsychiatric interview (M.I.N.I.): The development and validation of a structured diagnostic psychiatric interview for DSM-IV and ICD-10. The Journal of Clinical Psychiatry 59 Suppl 20, 22-33;quiz 34-57.

Stern, H. S. (2016). A test by any other name: $P$ values, Bayes factors, and statistical inference. Multivariate Behavioral Research, 51, 23-29.

Tadel, F., Baillet, S., Mosher, J. C., Pantazis, D., \& Leahy, R. M. (2011). Brainstorm: A user-friendly application for MEG/EEG analysis. Computational Intelligence and Neuroscience, 2011, 1-13.

Tamura, M., Spellman, T. J., Rosen, A. M., Gogos, J. A., \& Gordon, J. A. (2017). Hippocampal-prefrontal theta-gamma coupling during performance of a spatial working memory task. Nature Communications, 8, 2182.

Ter Braack, E. M., de Vos, C. C., \& van Putten, M. J. (2015). Masking the auditory evoked potential in TMS-EEG: A comparison of various methods. Brain Topography, 28, 520-528.

Thut, G., \& Pascual-Leone, A. (2010). A review of combined TMS-EEG studies to characterize lasting effects of repetitive TMS and assess their usefulness in cognitive and clinical neuroscience. Brain Topography, 22, 219-232. 
Tort, A. B., Komorowski, R. W., Manns, J. R., Kopell, N. J., \& Eichenbaum, H. (2009). Theta-gamma coupling increases during the learning of item-context associations. Proceedings of the National Academy of Sciences of the United States of America, 106, 20942-20947.

Turrigiano, G. (2011). Too many cooks? Intrinsic and synaptic homeostatic mechanisms in cortical circuit refinement. Annual Review of Neuroscience, 34, 89-103.

Turriziani, P., Smirni, D., Zappala, G., Mangano, G. R., Oliveri, M., \& Cipolotti, L. (2012). Enhancing memory performance with rTMS in healthy subjects and individuals with mild cognitive impairment: The role of the right dorsolateral prefrontal cortex. Frontiers in Human Neuroscience, 6, 62.

Verbruggen, F., Aron, A. R., Stevens, M. A., \& Chambers, C. D. (2010). Theta burst stimulation dissociates attention and action updating in human inferior frontal cortex. Proceedings of the National Academy of Sciences of the United States of America, 107, 13966-13971.

Viejo-Sobera, R., Redolar-Ripoll, D., Boixados, M., Palaus, M., Valero-Cabre, A., \& Marron, E. M. (2017). Impact of prefrontal theta burst stimulation on clinical neuropsychological tasks. Frontiers in Neuroscience, 11,462

Vogels, T. P., \& Abbott, L. F. (2009). Gating multiple signals through detailed balance of excitation and inhibition in spiking networks. Nature Neuroscience, 12, 483-491.

Vogels, T. P., Sprekeler, H., Zenke, F., Clopath, C., \& Gerstner, W. (2011). Inhibitory plasticity balances excitation and inhibition in sensory pathways and memory networks. Science, 334, 1569-1573.

Wagenmakers, E. J. (2007). A practical solution to the pervasive problems of p values. Psychonomic Bulletin \& Review, 14, 779-804.

Wagenmakers, E. J., Love, J., Marsman, M., Jamil, T., Ly, A., Verhagen, J., ... Morey, R. D. (2018a). Bayesian inference for psychology. Part II: Example applications with JASP. Psychonomic Bulletin \& Review, 25, 58-76.

Wagenmakers, E. J., Marsman, M., Jamil, T., Ly, A., Verhagen, J., Love, J., ... Morey, R. D. (2018b). Bayesian inference for psychology. Part I: Theoretical advantages and practical ramifications. Psychonomic Bulletin \& Review, 25, 35-57.

Watrous, A. J., Lee, D. J., Izadi, A., Gurkoff, G. G., Shahlaie, K., \& Ekstrom, A. D. (2013). A comparative study of human and rat hippocampal low-frequency oscillations during spatial navigation. Hippocampus, 23, 656-661.

Wiethoff, S., Hamada, M., \& Rothwell, J. C. (2014). Variability in response to transcranial direct current stimulation of the motor cortex. Brain Stimulation, 7, 468-475.

Wilcox, R. R. (1997). Introduction to robust estimation and hypothesis testing. San Diego, CA: Academic Press.

Wischnewski, M., \& Schutter, D. J. (2015). Efficacy and time course of theta burst stimulation in healthy humans. Brain Stimulation, 8, 685-692.

Wrase, J., Kahnt, T., Schlagenhauf, F., Beck, A., Cohen, M. X., Knutson, B., \& Heinz, A. (2007). Different neural systems adjust motor behavior in response to reward and punishment. Neurolmage, 36, 1253-1262.

Xue, M., Atallah, B. V., \& Scanziani, M. (2014). Equalizing excitation-inhibition ratios across visual cortical neurons. Nature, 511, 596-600.

Yizhar, O., Fenno, L. E., Prigge, M., Schneider, F., Davidson, T. J. ., O'Shea, D. J., ... Deisseroth, K. (2011). Neocortical excitation/inhibition balance in information processing and social dysfunction. Nature, 477, 171-178.

\section{SUPPORTING INFORMATION}

Additional supporting information may be found online in the Supporting Information section at the end of the article.

How to cite this article: Chung SW, Sullivan CM, Rogasch NC, et al. The effects of individualised intermittent theta burst stimulation in the prefrontal cortex: A TMS-EEG study. Hum Brain Mapp. 2019;40:608-627. https://doi.org/10. 1002/hbm.24398 